Supporting Informations for the Communication

\author{
Entitled
}

\title{
Silyl-substituted 1,4-Disila(Dewar-benzene): New Synthesis and Unexpected Insertion of CO into the Si-Si Bond to Form a Disilyl Ketone
}

Norio Nakata, Toru Oikawa, Takeshi Matsumoto, Yoshio Kabe, and Akira Sekiguchi*

Department of Chemistry, Graduate School of Pure and Applied Sciences,

University of Tsukuba, Tsukuba, Ibaraki 305-8571, Japan 


\section{Experimental Section}

General Procedure. All experiments were performed using high-vacuum line techniques or in an argon atmosphere of MBRAUN MB 150B-G glove-box. All solvents were predried over sodium benzophenone ketyl and finally dried and degassed over potassium mirror in vacuum prior to use. NMR spectra were recorded on a Bruker AC-300FT NMR spectrometer $\left({ }^{1} \mathrm{H}\right.$ NMR at $300.1 \mathrm{MHz} ;{ }^{13} \mathrm{C}$ NMR at 75.5 MHz; ${ }^{29} \mathrm{Si} \mathrm{NMR} \mathrm{at} \mathrm{59.6} \mathrm{MHz).} \mathrm{Mass-spectra} \mathrm{(direct} \mathrm{ionization)} \mathrm{were} \mathrm{obtained} \mathrm{on} \mathrm{JEOL}$ JMS SX-102 instrument. UV-spectra were recorded on a Shimadzu UV-3150 UV-Vis spectrophotometer. Infrared spectrum was obtained on a JASCO FT/IR-410 spectrophotometer. Preparative gel permeation liquid chromatography (GPLC) was performed on an LC-918 (Japan Analytical Industry Co., Ltd.) equipped with JAIGEL $1 \mathrm{H}$ and $2 \mathrm{H}$ columns (eluent: toluene).

\section{1,4-Bis(di-tert-butylmethylsilyl)-1,4-dichloro-2,3,5,6-tetraethyl-1,4-disilacyclohexa-2,5-}

diene (2). A solution of ${ }^{t} \mathrm{Bu}_{2} \mathrm{MeSiNa}^{1}(1.6 \mathrm{~g}, 8.9 \mathrm{mmol})$ in THF $(20 \mathrm{~mL})$ was added dropwise at room temperature to a solution of 1,1,4,4-tetrachloro-2,3,5,6-tetraethyl-1,4-disilacyclohexa-2,5$\operatorname{diene}^{2}(1.5 \mathrm{~g}, 4.1 \mathrm{mmol})$ in THF $(10 \mathrm{~mL})$, and the reaction mixture was stirred for $24 \mathrm{~h}$. After removal of the solvent in vacuo, the resulting sodium chloride was filtered through Celite and washed with hexane. The solvents were evaporated, and the residue was purified by recrystallization from hexane to give $2(0.2 \mathrm{~g}, 25 \%)$ as a colorless solid. Mp 296-297 ${ }^{\circ} \mathrm{C} .{ }^{1} \mathrm{H}$ NMR $\left(\mathrm{C}_{6} \mathrm{D}_{6}, \delta\right) 0.20$ (s, $6 \mathrm{H}), 1.14(\mathrm{~s}, 36 \mathrm{H}), 1.22\left(\mathrm{t},{ }^{3} J=7.1 \mathrm{~Hz}, 6 \mathrm{H}\right), 2.48\left(\mathrm{dq},{ }^{2} J=13.3 \mathrm{~Hz},{ }^{3} J=7.1 \mathrm{~Hz}, 4 \mathrm{H}\right), 2.56\left(\mathrm{dq},{ }^{2} J\right.$ $\left.=13.3 \mathrm{~Hz},{ }^{3} J=7.1 \mathrm{~Hz}, 4 \mathrm{H}\right) ;{ }^{13} \mathrm{C} \mathrm{NMR}\left(\mathrm{C}_{6} \mathrm{D}_{6}, \delta\right)-5.8(\mathrm{SiMe}), 14.9\left(\mathrm{CH}_{2} \mathrm{CH}_{3}\right), 22.1\left(\mathrm{CMe}_{3}\right), 26.6$ $\left(\mathrm{CH}_{2} \mathrm{CH}_{3}\right), 29.9\left(\mathrm{CMe}_{3}\right), 156.9(\mathrm{C}=\mathrm{C}) ;{ }^{29} \mathrm{Si} \mathrm{NMR}\left(\mathrm{C}_{6} \mathrm{D}_{6}, \delta\right)-5.3(\mathrm{Si}-\mathrm{C}), 2.3\left(\mathrm{SiMe}^{\mathrm{t}} \mathrm{Bu}_{2}\right) ; \mathrm{HRMS} \mathrm{m} / \mathrm{z}$ calcd for $\mathrm{C}_{30} \mathrm{H}_{62} \mathrm{Cl}_{2} \mathrm{Si}_{4}$ 604.3289, found 604.3306. Anal. Calcd for $\mathrm{C}_{30} \mathrm{H}_{62} \mathrm{Cl}_{2} \mathrm{Si}_{4}: \mathrm{C}, 59.45 ; \mathrm{H}, 10.31$. Found: C, 59.24; H, 10.25. 


\section{1,4-Bis(di-tert-butylmethylsilyl)-2,3,5,6-tetraethyl-1,4-disilabicyclo[2.2.0] hexa-2,5-diene}

(1). Dry oxygen-free THF $(2.5 \mathrm{~mL})$ was introduced into the mixture of $2(60 \mathrm{mg}, 0.1 \mathrm{mmol})$ and potassium graphite $(30 \mathrm{mg}, 0.2 \mathrm{mmol})$, and the reaction mixture was stirred for $2 \mathrm{~h}$ at room temperature. After removal of the solvent in vacuo, hexane was added. Filtration of the mixture followed by removal of the solvent in vacuo afforded $\mathbf{1}$ as a yellow solid. Finally, $\mathbf{1}$ was purified by recrystallization from hexane to give pale yellow crystals of $\mathbf{1}(37 \mathrm{mg}, 70 \%)$. Mp $108-110^{\circ} \mathrm{C} .{ }^{1} \mathrm{H}$ $\operatorname{NMR}\left(\mathrm{C}_{6} \mathrm{D}_{6}, \delta\right) 0.36(\mathrm{~s}, 6 \mathrm{H}), 1.06\left(\mathrm{t},{ }^{3} J=7.1 \mathrm{~Hz}, 12 \mathrm{H}\right), 1.15(\mathrm{~s}, 36 \mathrm{H}), 2.27\left(\mathrm{dq},{ }^{2} J=15.3 \mathrm{~Hz},{ }^{3} J=\right.$ $7.1 \mathrm{~Hz}, 4 \mathrm{H}), 2.59\left(\mathrm{dq},{ }^{2} J=15.3 \mathrm{~Hz},{ }^{3} J=7.1 \mathrm{~Hz}, 4 \mathrm{H}\right) ;{ }^{13} \mathrm{C} \mathrm{NMR}\left(\mathrm{C}_{6} \mathrm{D}_{6}, \delta\right)-5.5(\mathrm{Si} M e), 14.4$ $\left(\mathrm{CH}_{2} \mathrm{CH}_{3}\right), 20.7\left(\mathrm{CMe}_{3}\right), 26.9\left(\mathrm{CH}_{2} \mathrm{CH}_{3}\right), 29.8\left(\mathrm{CMe}_{3}\right), 168.4(\mathrm{C}=\mathrm{C}) ;{ }^{29} \mathrm{Si}$ NMR $\left(\mathrm{C}_{6} \mathrm{D}_{6}, \delta\right)-26.3$ (Si-C), $11.0\left(\mathrm{SiMe}^{\mathrm{t} B u_{2}}\right)$; UV (3-methylpentane) $\lambda_{\max } / \mathrm{nm}$ (ع) 335 (3000). HRMS $\mathrm{m} / \mathrm{z}$ calcd for $\mathrm{C}_{30} \mathrm{H}_{62} \mathrm{Si}_{4}$ 534.3942, found 534.3929.

\section{1,4-Bis(di-tert-butylmethylsilyl)-2,3,5,6-tetraethyl-1,4-disilabicyclo[2.2.1]hepta-2,5-dien-}

7-one (3). A THF (2 mL) solution of $1(54 \mathrm{mg}, 0.100 \mathrm{mmol})$ in a glass tube was irradiated with a high pressure $\mathrm{Hg}$ lamp (400 W, $\lambda>320 \mathrm{~nm}$ ) under a $\mathrm{CO}$ atmosphere for $1 \mathrm{~h}$. After removal of the solvent in vacuo, purification of the residue by gel permeation chromatography afforded $\mathbf{3}$ (40 mg, $71 \%)$ as yellow crystals. Mp $143-145{ }^{\circ} \mathrm{C} .{ }^{1} \mathrm{H} \mathrm{NMR}\left(\mathrm{C}_{6} \mathrm{D}_{6}, \delta\right) 0.30(\mathrm{~s}, 6 \mathrm{H}), 1.03\left(\mathrm{t},{ }^{3} \mathrm{~J}=7.4 \mathrm{~Hz}, 12 \mathrm{H}\right)$, $1.15(\mathrm{~s}, 36 \mathrm{H}), 2.46\left(\mathrm{dq},{ }^{2} J=13.2 \mathrm{~Hz},{ }^{3} J=7.4 \mathrm{~Hz}, 4 \mathrm{H}\right), 2.91\left(\mathrm{dq},{ }^{2} J=13.2 \mathrm{~Hz},{ }^{3} J=7.4 \mathrm{~Hz}, 4 \mathrm{H}\right) ;{ }^{13} \mathrm{C}$ $\operatorname{NMR}\left(\mathrm{C}_{6} \mathrm{D}_{6}, \delta\right) 1.2(\mathrm{SiMe}), 17.3\left(\mathrm{CH}_{2} \mathrm{CH}_{3}\right), 20.6\left(\mathrm{CMe}_{3}\right), 25.9\left(\mathrm{CH}_{2} \mathrm{CH}_{3}\right), 29.4\left(\mathrm{CMe}_{3}\right), 159.5(\mathrm{C}=\mathrm{C})$, $260.3(C=\mathrm{O}) ;{ }^{29} \mathrm{Si}$ NMR $\left.\left(\mathrm{C}_{6} \mathrm{D}_{6}, \delta\right)-45.3(\mathrm{Si}-\mathrm{C}), 8.8\left(\mathrm{SiMe}^{\mathrm{t} B u}\right)_{2}\right)$ IR $(\mathrm{KBr}): v(\mathrm{C}=\mathrm{O}) 1673 \mathrm{~cm}^{-1}$. UV/Vis (hexane) $\lambda_{\max } / \mathrm{nm}(\varepsilon) 291$ (12600), 431 (50). HRMS m/z calcd for $\mathrm{C}_{31} \mathrm{H}_{62} \mathrm{OSi}_{4}$ 562.3878, found 562.3860 . 


\section{2,5-Bis(di-tert-butylmethylsilyl)-1,3,4,6-tetraethyl-2,5-disilatricyclo[3.1.0.0 $\left.{ }^{2,6}\right]$ hex-3-ene}

(4). A cyclohexane- $d_{12}(0.5 \mathrm{~mL})$ solution of $\mathbf{1}(14 \mathrm{mg}, 0.026 \mathrm{mmol})$ in a sealed NMR tube was irradiated with a high pressure $\mathrm{Hg}$ lamp $(400 \mathrm{~W}, \lambda>320 \mathrm{~nm}$ ) for $6 \mathrm{~h}$. The solvent was evaporated to give pure 4 as a pale yellow oil in quantitative yield. ${ }^{1} \mathrm{H} N M R\left(\mathrm{C}_{6} \mathrm{D}_{6}, \delta\right) 0.14(\mathrm{~s}, 6 \mathrm{H}), 0.95(\mathrm{t}, J=7.4$ $\mathrm{Hz}, 6 \mathrm{H}), 1.18(\mathrm{t}, 36 \mathrm{H}), 1.36\left(\mathrm{t},{ }^{3} J=7.1 \mathrm{~Hz}, 6 \mathrm{H}\right), 1.89\left(\mathrm{q},{ }^{3} J=7.1 \mathrm{~Hz}, 4 \mathrm{H}\right), 2.59\left(\mathrm{q},{ }^{3} J=7.1 \mathrm{~Hz}, 4\right.$ H). ${ }^{13} \mathrm{C}$ NMR $\left(\mathrm{C}_{6} \mathrm{D}_{6}, \delta\right)-4.1(\mathrm{SiMe}), 17.7\left(\mathrm{CH}_{2} \mathrm{CH}_{3}\right), 19.0\left(\mathrm{CH}_{2} \mathrm{CH}_{3}\right), 22.3\left(\mathrm{CMe}_{3}\right), 24.5\left(\mathrm{CH}_{2} \mathrm{CH}_{3}\right)$, $25.3\left(\mathrm{CH}_{2} \mathrm{CH}_{3}\right), 31.2\left(\mathrm{CMe}_{3}\right), 66.9(\mathrm{C}-\mathrm{C}), 161.7(\mathrm{C}=\mathrm{C}) ;{ }^{29} \mathrm{Si}$ NMR $\left(\mathrm{C}_{6} \mathrm{D}_{6}, \delta\right)-100.3(\mathrm{Si}-\mathrm{C}), 14.4$ $\left(\mathrm{SiMe}^{t} \mathrm{Bu}_{2}\right) ;$ HRMS $m / z$ calcd for $\mathrm{C}_{30} \mathrm{H}_{62} \mathrm{Si}_{4} 534.3942$, found 534.3929 .

\section{References}

(1) Sekiguchi, A.; Fukawa, T.; Nakamoto, M.; Lee, V. Ya.; Ichinohe, M. J. Am. Chem. Soc. 2002, 124,9865 .

(2) Kang, S. H.; Han, J. S.; Lee, M. E.; Yoo, B. R.; Jung, I. N. Organometallics 2003, 22, 2551. 
Table 1. Crystal data and structure refinement for 1,4-disila(Dewer-benzene).

Identification code

Empirical formula

Formula weight

Temperature

Wavelength

Crystal system

Space group

Unit cell dimensions

Volume

Z

Density (calculated)

Absorption coefficient

$\mathrm{F}(000)$

Crystal size

Theta range for data collection

Index ranges

Reflections collected

Independent reflections

Completeness to theta $=25.00^{\circ}$

Refinement method

Data / restraints / parameters

Goodness-of-fit on $\mathrm{F}^{2}$

Final $R$ indices [I $>2 \operatorname{sigma}(\mathrm{I})]$

$\mathrm{R}$ indices (all data)

Largest diff. peak and hole
1,4-disila(Dewer-benzene)

C30 H62 Si4

535.16

$120(2) \mathrm{K}$

$0.71070 \AA$

monoclinic

$P 2_{1} / \mathrm{c}$

$a=16.5790(16) \AA \quad \alpha=90^{\circ}$.

$b=9.7770(10) \AA \quad \beta=95.1200(6)^{\circ}$.

$c=42.188(2) \AA \quad \gamma=90^{\circ}$.

6811.1(10) $\AA^{3}$

8

$1.044 \mathrm{Mg} / \mathrm{m}^{3}$

$0.191 \mathrm{~mm}^{-1}$

2384

$0.40 \times 0.30 \times 0.10 \mathrm{~mm}^{3}$

2.14 to $25.00^{\circ}$.

$0<=\mathrm{h}<=19,0<=\mathrm{k}<=11,-47<=\mathrm{l}<=44$

10937

$10937[R($ int $)=0.070]$

$91.1 \%$

Full-matrix least-squares on $\mathrm{F}^{2}$

10937 / 0 / 649

1.063

$R_{1}=0.0669, \mathrm{w} R_{2}=0.1761$

$R_{1}=0.1015, \mathrm{w} R_{2}=0.2019$

0.843 and -0.457 e. $\AA^{-3}$ 


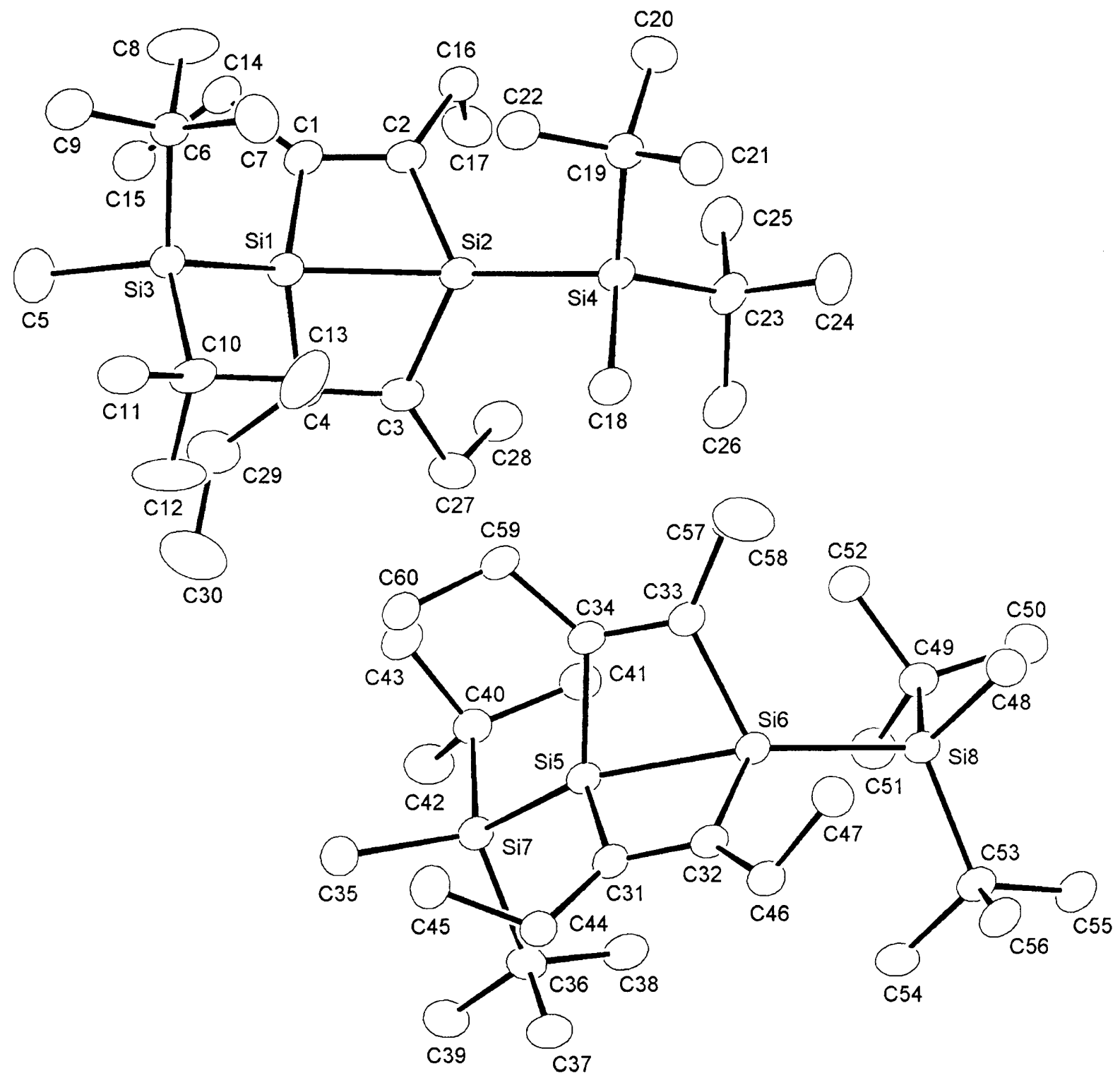

Figure 1. ORTEP drawing of 1,4-bis(di-tert-butylmethylsilyl)-2,3,5,6-tetraethyl-1,4disilabicyclo[2.2.0]hexa-2,5-diene (2) (30\% probability) with nonhydrogen atoms. 
Table 2. Atomic coordinates ( $\left.\mathrm{x} 10^{4}\right)$ and equivalent isotropic displacement parameters $\left(\AA^{2} \mathrm{x}\right.$ $10^{3}$ ) for 1,4-disila(Dewer-benzene). $\mathrm{U}(\mathrm{eq})$ is defined as one third of the trace of the orthogonalized $\mathrm{U}^{\mathrm{ij}}$ tensor.

\begin{tabular}{|c|c|c|c|c|}
\hline & $\mathrm{x}$ & $\mathrm{y}$ & z & $\mathrm{U}(\mathrm{eq})$ \\
\hline $\operatorname{Si}(1)$ & $5580(1)$ & $1548(1)$ & $1552(1)$ & $36(1)$ \\
\hline$C(1)$ & $4683(2)$ & $663(4)$ & $1315(1)$ & $40(1)$ \\
\hline$C(2)$ & $4949(2)$ & $427(4)$ & $1025(1)$ & $36(1)$ \\
\hline $\operatorname{Si}(2)$ & $6055(1)$ & $1062(1)$ & 1071(1) & $35(1)$ \\
\hline$C(3)$ & $6632(2)$ & $-180(4)$ & $1368(1)$ & $41(1)$ \\
\hline$C(4)$ & $6328(2)$ & $91(4)$ & $1649(1)$ & $43(1)$ \\
\hline $\operatorname{Si}(3)$ & $5404(1)$ & $3363(1)$ & $1915(1)$ & $36(1)$ \\
\hline$C(5)$ & $5014(3)$ & $2571(5)$ & $2279(1)$ & $64(1)$ \\
\hline$C(6)$ & $4595(2)$ & $4632(4)$ & $1750(1)$ & $41(1)$ \\
\hline$C(7)$ & $4917(3)$ & $5712(5)$ & $1523(1)$ & $65(1)$ \\
\hline$C(8)$ & $3908(3)$ & $3845(6)$ & $1571(2)$ & $83(2)$ \\
\hline $\mathrm{C}(9)$ & $4251(3)$ & $5456(5)$ & $2018(1)$ & $59(1)$ \\
\hline $\mathrm{C}(10)$ & $6442(2)$ & $4148(5)$ & $2034(1)$ & $50(1)$ \\
\hline$C(11)$ & $6388(3)$ & $5373(5)$ & $2256(1)$ & $55(1)$ \\
\hline$C(12)$ & $6967(3)$ & $3049(6)$ & $2215(2)$ & $90(2)$ \\
\hline$C(13)$ & $6860(3)$ & $4595(7)$ & $1743(1)$ & $85(2)$ \\
\hline$C(14)$ & $3898(2)$ & $150(5)$ & $1435(1)$ & $43(1)$ \\
\hline$C(15)$ & $4037(2)$ & $-551(5)$ & $1753(1)$ & $50(1)$ \\
\hline$C(16)$ & $4462(2)$ & $-311(4)$ & $754(1)$ & $41(1)$ \\
\hline$C(17)$ & $4597(3)$ & $-1841(5)$ & $752(1)$ & $57(1)$ \\
\hline $\mathrm{Si}(4)$ & $6682(1)$ & $2233(1)$ & $665(1)$ & $39(1)$ \\
\hline$C(18)$ & $7614(2)$ & $3048(5)$ & $870(1)$ & $49(1)$ \\
\hline$C(19)$ & $5980(2)$ & $3671(4)$ & $503(1)$ & $43(1)$ \\
\hline$C(20)$ & $5276(3)$ & $3155(5)$ & $265(1)$ & $58(1)$ \\
\hline$C(21)$ & $6449(3)$ & $4791(5)$ & $340(1)$ & $56(1)$ \\
\hline $\mathrm{C}(22)$ & $5597(3)$ & $4331(4)$ & $784(1)$ & $48(1)$ \\
\hline$C(23)$ & $7001(3)$ & $987(5)$ & $351(1)$ & $48(1)$ \\
\hline
\end{tabular}




$\begin{array}{lrrrr}\mathrm{C}(24) & 7302(3) & 1738(6) & 60(1) & 63(1) \\ \mathrm{C}(25) & 6304(3) & 9(5) & 237(1) & 57(1) \\ \mathrm{C}(26) & 7708(3) & 116(5) & 500(1) & 59(1) \\ \mathrm{C}(27) & 7199(3) & -1359(5) & 1310(1) & 51(1) \\ \mathrm{C}(28) & 6821(3) & -2430(5) & 1082(1) & 53(1) \\ \mathrm{C}(29) & 6417(3) & -833(5) & 1953(1) & 64(1) \\ \mathrm{C}(30) & 7153(4) & -672(7) & 2166(2) & 93(2) \\ \mathrm{Si}(5) & 10627(1) & 4139(1) & 1549(1) & 34(1) \\ \mathrm{C}(31) & 11498(2) & 5431(4) & 1630(1) & 38(1) \\ \mathrm{C}(32) & 11694(2) & 5808(4) & 1338(1) & 36(1) \\ \mathrm{Si}(6) & 10978(1) & 4711(1) & 1051(1) & 34(1) \\ \mathrm{C}(33) & 9914(2) & 5484(4) & 1066(1) & 42(1) \\ \mathrm{C}(34) & 9715(2) & 5180(4) & 1361(1) & 38(1) \\ \mathrm{Si}(7) & 10462(1) & 2187(1) & 1874(1) & 37(1) \\ \mathrm{C}(35) & 10297(3) & 2695(5) & 2295(1) & 48(1) \\ \mathrm{C}(36) & 11451(2) & 1166(4) & 1897(1) & 39(1) \\ \mathrm{C}(37) & 12162(2) & 2164(5) & 1962(1) & 49(1) \\ \mathrm{C}(38) & 11563(2) & 416(5) & 1586(1) & 52(1) \\ \mathrm{C}(39) & 1149(5) & 2172(1) & 54(1) \\ \mathrm{C}(40) & 11495(3) & 122(5) \\ \mathrm{C}(41) & 9522(2) & 1202(4) & 1699(1) & 40(1) \\ \mathrm{C}(42) & 9492(2) & 1127(5) & 1334(1) & 47(1) \\ \mathrm{C}(43) & 9484(3) & -264(5) & 1835(1) & 57(1) \\ \mathrm{C}(44) & 10639(2) & 2329(4) & 429(1) & 41(1) \\ \mathrm{C}(45) & 19645(3) & 2028(5) & 72(1) & 48(1) \\ \mathrm{C}(46) & 1964(5) & 1785(1) & 51(1) \\ \mathrm{C}(47) & 673(2) & 6002(5) & 1946(1) & 42(1) \\ \mathrm{Si}(8) & 11269(3) & 6258(5) & 2189(1) & 54(1) \\ \mathrm{C}(48) & 12288(2) & 6920(4) & 1266(1) & 38(1) \\ \mathrm{C}(49) & 11989(3) & 7819(5) & 985(1) & 49(1) \\ \mathrm{C}(50) & 11353(1) & 3815(1) & 561(1) & 35(1) \\ & 11182(2) & 5186(4) & 249(1) & 49(1)\end{array}$




$\begin{array}{lrrrr}\mathrm{C}(53) & 12477(2) & 3354(4) & 599(1) & 41(1) \\ \mathrm{C}(54) & 12706(3) & 2519(5) & 904(1) & 52(1) \\ \mathrm{C}(55) & 12719(3) & 2524(5) & 313(1) & 53(1) \\ \mathrm{C}(56) & 12974(2) & 4681(5) & 618(1) & 51(1) \\ \mathrm{C}(57) & 9391(3) & 6312(5) & 823(1) & 54(1) \\ \mathrm{C}(58) & 9631(4) & 7751(6) & 823(2) & 81(2) \\ \mathrm{C}(59) & 8958(2) & 5689(5) & 1505(1) & 46(1) \\ \mathrm{C}(60) & 9075(2) & 6003(5) & 1855(1) & 44(1)\end{array}$


Table 3. Bond lengths $[\AA]$ and angles $\left[{ }^{\circ}\right]$ for 1,4 -disila(Dewer-benzene).

\begin{tabular}{|c|c|c|c|}
\hline $\operatorname{Si}(1)-C(4)$ & $1.909(4)$ & $C(23)-C(25)$ & $1.544(6)$ \\
\hline $\operatorname{Si}(1)-C(1)$ & $1.921(4)$ & $C(23)-C(24)$ & $1.550(6)$ \\
\hline $\operatorname{Si}(1)-\operatorname{Si}(2)$ & $2.2908(15)$ & $\mathrm{C}(27)-\mathrm{C}(28)$ & $1.518(6)$ \\
\hline $\operatorname{Si}(1)-\operatorname{Si}(3)$ & $2.3798(16)$ & $C(29)-C(30)$ & $1.458(7)$ \\
\hline$C(1)-C(2)$ & $1.356(6)$ & $\operatorname{Si}(5)-C(31)$ & $1.926(4)$ \\
\hline$C(1)-C(14)$ & $1.524(5)$ & $\operatorname{Si}(5)-C(34)$ & $1.932(4)$ \\
\hline$C(2)-C(16)$ & $1.521(5)$ & $\operatorname{Si}(5)-\operatorname{Si}(6)$ & $2.2969(15)$ \\
\hline$C(2)-\operatorname{Si}(2)$ & $1.928(4)$ & $\operatorname{Si}(5)-\operatorname{Si}(7)$ & $2.3807(15)$ \\
\hline $\operatorname{Si}(2)-C(3)$ & $1.935(4)$ & $C(31)-C(32)$ & $1.353(6)$ \\
\hline $\operatorname{Si}(2)-\operatorname{Si}(4)$ & $2.3764(16)$ & $C(31)-C(44)$ & $1.521(5)$ \\
\hline$C(3)-C(4)$ & $1.356(6)$ & $C(32)-C(46)$ & $1.517(5)$ \\
\hline$C(3)-C(27)$ & $1.522(6)$ & $\mathrm{C}(32)-\mathrm{Si}(6)$ & $1.941(4)$ \\
\hline$C(4)-C(29)$ & $1.566(6)$ & $\mathrm{Si}(6)-\mathrm{C}(33)$ & $1.925(4)$ \\
\hline $\operatorname{Si}(3)-C(5)$ & $1.884(5)$ & $\operatorname{Si}(6)-\operatorname{Si}(8)$ & $2.3797(15)$ \\
\hline $\operatorname{Si}(3)-C(10)$ & $1.911(4)$ & $C(33)-C(34)$ & $1.349(6)$ \\
\hline $\operatorname{Si}(3)-C(6)$ & $1.911(4)$ & $C(33)-C(57)$ & $1.516(6)$ \\
\hline$C(6)-C(8)$ & $1.518(6)$ & $C(34)-C(59)$ & $1.527(5)$ \\
\hline$C(6)-C(9)$ & $1.540(6)$ & $\mathrm{Si}(7)-\mathrm{C}(35)$ & $1.889(4)$ \\
\hline$C(6)-C(7)$ & $1.551(6)$ & $\operatorname{Si}(7)-C(36)$ & $1.915(4)$ \\
\hline $\mathrm{C}(10)-\mathrm{C}(13)$ & $1.524(7)$ & $\mathrm{Si}(7)-C(40)$ & $1.921(4)$ \\
\hline$C(10)-C(11)$ & $1.530(6)$ & $C(36)-C(38)$ & $1.528(6)$ \\
\hline$C(10)-C(12)$ & $1.543(7)$ & $C(36)-C(37)$ & $1.536(5)$ \\
\hline$C(14)-C(15)$ & $1.508(6)$ & $\mathrm{C}(36)-\mathrm{C}(39)$ & $1.541(6)$ \\
\hline$C(16)-C(17)$ & $1.512(6)$ & $\mathrm{C}(40)-\mathrm{C}(43)$ & $1.540(6)$ \\
\hline $\operatorname{Si}(4)-C(18)$ & $1.880(4)$ & $C(40)-C(41)$ & $1.535(6)$ \\
\hline $\operatorname{Si}(4)-C(23)$ & $1.909(4)$ & $C(40)-C(42)$ & $1.546(6)$ \\
\hline $\mathrm{Si}(4)-\mathrm{C}(19)$ & $1.912(4)$ & $C(44)-C(45)$ & $1.507(6)$ \\
\hline$C(19)-C(22)$ & $1.536(6)$ & $C(46)-C(47)$ & $1.520(6)$ \\
\hline$C(19)-C(21)$ & $1.540(6)$ & $\mathrm{Si}(8)-\mathrm{C}(48)$ & $1.881(4)$ \\
\hline$C(19)-C(20)$ & $1.553(6)$ & $\mathrm{Si}(8)-\mathrm{C}(53)$ & $1.911(4)$ \\
\hline$C(23)-C(26)$ & $1.538(6)$ & $\operatorname{Si}(8)-C(49)$ & $1.924(4)$ \\
\hline
\end{tabular}




\begin{tabular}{|c|c|c|c|}
\hline$C(49)-C(50)$ & $1.538(6)$ & $C(5)-\operatorname{Si}(3)-C(10)$ & $108.6(2)$ \\
\hline$C(49)-C(51)$ & $1.542(6)$ & $C(5)-S i(3)-C(6)$ & $106.4(2)$ \\
\hline$C(49)-C(52)$ & $1.543(5)$ & $C(10)-\operatorname{Si}(3)-C(6)$ & $114.9(2)$ \\
\hline$C(53)-C(56)$ & $1.536(6)$ & $C(5)-\operatorname{Si}(3)-\operatorname{Si}(1)$ & $106.80(17)$ \\
\hline$C(53)-C(55)$ & $1.539(6)$ & $C(10)-\operatorname{Si}(3)-\operatorname{Si}(1)$ & $107.99(14)$ \\
\hline$C(53)-C(54)$ & $1.542(6)$ & $C(6)-\operatorname{Si}(3)-\operatorname{Si}(1)$ & $111.81(13)$ \\
\hline$C(57)-C(58)$ & $1.463(7)$ & $C(8)-C(6)-C(9)$ & $108.6(4)$ \\
\hline \multirow[t]{2}{*}{$C(59)-C(60)$} & $1.503(6)$ & $C(8)-C(6)-C(7)$ & $108.9(4)$ \\
\hline & & $C(9)-C(6)-C(7)$ & $105.5(4)$ \\
\hline$C(4)-\operatorname{Si}(1)-C(1)$ & $103.48(18)$ & $C(8)-C(6)-S i(3)$ & $108.8(3)$ \\
\hline$C(4)-S i(1)-S i(2)$ & $76.55(14)$ & $C(9)-C(6)-S i(3)$ & $111.4(3)$ \\
\hline$C(1)-\operatorname{Si}(1)-\operatorname{Si}(2)$ & $75.92(13)$ & $C(7)-C(6)-S i(3)$ & $113.5(3)$ \\
\hline $\mathrm{C}(4)-\mathrm{Si}(1)-\mathrm{Si}(3)$ & $122: 17(14)$ & $C(13)-C(10)-C(11)$ & $109.0(4)$ \\
\hline$C(1)-\operatorname{Si}(1)-\operatorname{Si}(3)$ & $122.52(13)$ & $C(13)-C(10)-C(12)$ & $108.8(5)$ \\
\hline $\operatorname{Si}(2)-\operatorname{Si}(1)-\operatorname{Si}(3)$ & $142.82(6)$ & $C(11)-C(10)-C(12)$ & $107.5(4)$ \\
\hline $\mathrm{C}(2)-\mathrm{C}(1)-\mathrm{C}(14)$ & $126.9(4)$ & $C(13)-C(10)-\operatorname{Si}(3)$ & $111.6(3)$ \\
\hline$C(2)-C(1)-S i(1)$ & $104.3(3)$ & $C(11)-C(10)-S i(3)$ & $112.0(3)$ \\
\hline$C(14)-C(1)-S i(1)$ & $128.3(3)$ & $C(12)-C(10)-S i(3)$ & $107.8(3)$ \\
\hline$C(1)-C(2)-C(16)$ & $124.3(3)$ & $C(15)-C(14)-C(1)$ & $112.2(3)$ \\
\hline$C(1)-C(2)-S i(2)$ & $103.7(3)$ & $C(17)-C(16)-C(2)$ & $113.9(3)$ \\
\hline$C(16)-C(2)-\operatorname{Si}(2)$ & $131.7(3)$ & $C(18)-\operatorname{Si}(4)-C(23)$ & $108.9(2)$ \\
\hline$C(2)-S i(2)-C(3)$ & $105.90(17)$ & $C(18)-\operatorname{Si}(4)-C(19)$ & $107.6(2)$ \\
\hline$C(2)-\operatorname{Si}(2)-\operatorname{Si}(1)$ & $75.92(12)$ & $C(23)-\operatorname{Si}(4)-C(19)$ & $114.7(2)$ \\
\hline $\mathrm{C}(3)-\mathrm{Si}(2)-\mathrm{Si}(1)$ & $75.29(13)$ & $\mathrm{C}(18)-\mathrm{Si}(4)-\mathrm{Si}(2)$ & $105.47(15)$ \\
\hline$C(2)-\operatorname{Si}(2)-\operatorname{Si}(4)$ & $123.80(13)$ & $C(23)-\operatorname{Si}(4)-\operatorname{Si}(2)$ & $111.02(15)$ \\
\hline$C(3)-\operatorname{Si}(2)-\operatorname{Si}(4)$ & $123.08(13)$ & $C(19)-\operatorname{Si}(4)-\operatorname{Si}(2)$ & $108.68(13)$ \\
\hline $\operatorname{Si}(1)-\operatorname{Si}(2)-\operatorname{Si}(4)$ & $138.21(6)$ & $C(22)-C(19)-C(21)$ & $107.9(4)$ \\
\hline$C(4)-C(3)-C(27)$ & $124.9(4)$ & $C(22)-C(19)-C(20)$ & $107.2(3)$ \\
\hline $\mathrm{C}(4)-\mathrm{C}(3)-\mathrm{Si}(2)$ & $104.1(3)$ & $C(21)-C(19)-C(20)$ & $108.9(4)$ \\
\hline$C(27)-C(3)-\operatorname{Si}(2)$ & $130.4(3)$ & $\mathrm{C}(22)-\mathrm{C}(19)-\mathrm{Si}(4)$ & $108.3(3)$ \\
\hline$C(3)-C(4)-C(29)$ & $126.1(4)$ & $\mathrm{C}(21)-\mathrm{C}(19)-\mathrm{Si}(4)$ & $111.5(3)$ \\
\hline$C(3)-C(4)-S i(1)$ & $104.1(3)$ & $C(20)-C(19)-\operatorname{Si}(4)$ & $113.0(3)$ \\
\hline$C(29)-C(4)-S i(1)$ & $128.3(3)$ & $C(26)-C(23)-C(25)$ & $107.9(4)$ \\
\hline
\end{tabular}




\begin{tabular}{|c|c|c|c|}
\hline$C(26)-C(23)-C(24)$ & $107.4(4)$ & $C(36)-S i(7)-C(40)$ & $114.85(18)$ \\
\hline$C(25)-C(23)-C(24)$ & $109.4(4)$ & $C(35)-\operatorname{Si}(7)-\operatorname{Si}(5)$ & $111.40(15)$ \\
\hline$C(26)-C(23)-S i(4)$ & $108.5(3)$ & $\mathrm{C}(36)-\mathrm{Si}(7)-\mathrm{Si}(5)$ & $107.78(13)$ \\
\hline$C(25)-C(23)-S i(4)$ & $111.4(3)$ & $\mathrm{C}(40)-\mathrm{Si}(7)-\mathrm{Si}(5)$ & $108.14(13)$ \\
\hline $\mathrm{C}(24)-\mathrm{C}(23)-\mathrm{Si}(4)$ & $112.0(3)$ & $C(38)-C(36)-C(37)$ & $108.0(4)$ \\
\hline$C(3)-C(27)-C(28)$ & $113.5(3)$ & $\mathrm{C}(38)-\mathrm{C}(36)-\mathrm{C}(39)$ & $109.0(4)$ \\
\hline$C(30)-C(29)-C(4)$ & $117.1(5)$ & $C(37)-C(36)-C(39)$ & $107.7(3)$ \\
\hline $\mathrm{C}(31)-\mathrm{Si}(5)-\mathrm{C}(34)$ & $105.90(17)$ & $C(38)-C(36)-S i(7)$ & $112.0(3)$ \\
\hline$C(31)-\operatorname{Si}(5)-\operatorname{Si}(6)$ & $75.90(13)$ & $C(37)-C(36)-S i(7)$ & $108.6(3)$ \\
\hline$C(34)-\operatorname{Si}(5)-\operatorname{Si}(6)$ & $75.54(12)$ & $\mathrm{C}(39)-\mathrm{C}(36)-\mathrm{Si}(7)$ & $111.5(3)$ \\
\hline$C(31)-\operatorname{Si}(5)-\operatorname{Si}(7)$ & $123.06(13)$ & $C(43)-C(40)-C(41)$ & $107.8(3)$ \\
\hline$C(34)-\operatorname{Si}(5)-\operatorname{Si}(7)$ & $122.19(13)$ & $\mathrm{C}(43)-\mathrm{C}(40)-\mathrm{C}(42)$ & $107.4(3)$ \\
\hline $\operatorname{Si}(6)-\operatorname{Si}(5)-\operatorname{Si}(7)$ & $140.67(6)$ & $C(41)-C(40)-C(42)$ & $109.1(4)$ \\
\hline$C(32)-C(31)-C(44)$ & $126.0(4)$ & $\mathrm{C}(43)-\mathrm{C}(40)-\mathrm{Si}(7)$ & $108.7(3)$ \\
\hline $\mathrm{C}(32)-\mathrm{C}(31)-\mathrm{Si}(5)$ & $104.6(3)$ & $C(41)-C(40)-\operatorname{Si}(7)$ & $111.2(3)$ \\
\hline$C(44)-C(31)-\operatorname{Si}(5)$ & 129.3(3) & $\mathrm{C}(42)-\mathrm{C}(40)-\mathrm{Si}(7)$ & $112.4(3)$ \\
\hline $\mathrm{C}(31)-\mathrm{C}(32)-\mathrm{C}(46)$ & $126.3(4)$ & $C(45)-C(44)-C(31)$ & $114.8(3)$ \\
\hline$C(31)-C(32)-S i(6)$ & $103.6(3)$ & $\mathrm{C}(32)-\mathrm{C}(46)-\mathrm{C}(47)$ & $113.4(3)$ \\
\hline$C(46)-C(32)-\operatorname{Si}(6)$ & $129.9(3)$ & $C(48)-\operatorname{Si}(8)-C(53)$ & $108.19(19)$ \\
\hline$C(33)-\operatorname{Si}(6)-C(32)$ & $105.88(17)$ & $\mathrm{C}(48)-\mathrm{Si}(8)-\mathrm{C}(49)$ & $106.66(19)$ \\
\hline$C(33)-\operatorname{Si}(6)-\operatorname{Si}(5)$ & $75.98(13)$ & $C(53)-\operatorname{Si}(8)-C(49)$ & $114.64(18)$ \\
\hline $\mathrm{C}(32)-\mathrm{Si}(6)-\mathrm{Si}(5)$ & $75.83(12)$ & $C(48)-\operatorname{Si}(8)-\operatorname{Si}(6)$ & $108.08(15)$ \\
\hline$C(33)-\operatorname{Si}(6)-\operatorname{Si}(8)$ & $118.91(13)$ & $\mathrm{C}(53)-\mathrm{Si}(8)-\mathrm{Si}(6)$ & $109.94(13)$ \\
\hline $\mathrm{C}(32)-\mathrm{Si}(6)-\mathrm{Si}(8)$ & $123.63(12)$ & $\mathrm{C}(49)-\mathrm{Si}(8)-\mathrm{Si}(6)$ & $109.09(13)$ \\
\hline $\operatorname{Si}(5)-\operatorname{Si}(6)-\operatorname{Si}(8)$ & $144.28(6)$ & $C(50)-C(49)-C(51)$ & $109.0(4)$ \\
\hline $\mathrm{C}(34)-\mathrm{C}(33)-\mathrm{C}(57)$ & $124.7(4)$ & $\mathrm{C}(50)-\mathrm{C}(49)-\mathrm{C}(52)$ & $107.7(3)$ \\
\hline$C(34)-C(33)-\operatorname{Si}(6)$ & $104.1(3)$ & $C(51)-C(49)-C(52)$ & 107.4(3) \\
\hline$C(57)-C(33)-\operatorname{Si}(6)$ & $131.2(3)$ & $\mathrm{C}(50)-\mathrm{C}(49)-\mathrm{Si}(8)$ & $111.7(3)$ \\
\hline $\mathrm{C}(33)-\mathrm{C}(34)-\mathrm{C}(59)$ & $125.0(4)$ & $C(51)-C(49)-\operatorname{Si}(8)$ & $112.3(3)$ \\
\hline$C(33)-C(34)-\operatorname{Si}(5)$ & $104.3(3)$ & $\mathrm{C}(52)-\mathrm{C}(49)-\mathrm{Si}(8)$ & $108.6(3)$ \\
\hline$C(59)-C(34)-\operatorname{Si}(5)$ & $130.5(3)$ & $\mathrm{C}(56)-\mathrm{C}(53)-\mathrm{C}(55)$ & $108.0(3)$ \\
\hline$C(35)-\operatorname{Si}(7)-C(36)$ & $106.45(19)$ & $\mathrm{C}(56)-\mathrm{C}(53)-\mathrm{C}(54)$ & $108.3(3)$ \\
\hline$C(35)-\operatorname{Si}(7)-C(40)$ & $108.26(19)$ & $\mathrm{C}(55)-\mathrm{C}(53)-\mathrm{C}(54)$ & $108.3(4)$ \\
\hline
\end{tabular}




$\begin{array}{llll}\mathrm{C}(56)-\mathrm{C}(53)-\mathrm{Si}(8) & 108.7(3) & \mathrm{C}(58)-\mathrm{C}(57)-\mathrm{C}(33) & 112.0(4) \\ \mathrm{C}(55)-\mathrm{C}(53)-\mathrm{Si}(8) & 112.3(3) & \mathrm{C}(60)-\mathrm{C}(59)-\mathrm{C}(34) & 114.8(3) \\ \mathrm{C}(54)-\mathrm{C}(53)-\mathrm{Si}(8) & 111.2(3) & & \end{array}$

Symmetry transformations used to generate equivalent atoms: 
Table 4. Anisotropic displacement parameters $\left(\AA^{2} \times 10^{3}\right)$ for 1,4-disila(Dewer-benzene). The anisotropic displacement factor exponent takes the form: $-2 \pi^{2}\left[h^{2} a^{* 2} U^{11}+\ldots+2 h k a^{*} b^{*}\right.$ $\left.\mathrm{U}^{12}\right]$

\begin{tabular}{|c|c|c|c|c|c|c|}
\hline & $\mathrm{U}^{11}$ & $U^{22}$ & $\mathrm{U}^{33}$ & $\mathrm{U}^{23}$ & $\mathrm{U}^{13}$ & $\mathrm{U}^{12}$ \\
\hline $\operatorname{Si}(1)$ & $29(1)$ & $39(1)$ & $39(1)$ & $0(1)$ & $2(1)$ & $-3(1)$ \\
\hline$C(1)$ & $30(2)$ & $42(2)$ & $49(3)$ & $3(2)$ & $6(2)$ & $-2(2)$ \\
\hline$C(2)$ & $30(2)$ & $35(2)$ & $43(2)$ & $1(2)$ & $1(2)$ & $0(2)$ \\
\hline $\operatorname{Si}(2)$ & $27(1)$ & $39(1)$ & $40(1)$ & $-1(1)$ & $3(1)$ & $2(1)$ \\
\hline$C(3)$ & $32(2)$ & $36(2)$ & $52(3)$ & $-5(2)$ & $-4(2)$ & $2(2)$ \\
\hline$C(4)$ & $41(2)$ & $35(2)$ & $51(3)$ & $6(2)$ & $-6(2)$ & $0(2)$ \\
\hline $\operatorname{Si}(3)$ & $30(1)$ & $40(1)$ & $38(1)$ & $0(1)$ & $3(1)$ & $-1(1)$ \\
\hline$C(5)$ & $83(4)$ & $52(3)$ & $61(3)$ & $10(2)$ & $28(3)$ & $7(3)$ \\
\hline$C(6)$ & $31(2)$ & $48(3)$ & $44(3)$ & $-3(2)$ & $0(2)$ & $1(2)$ \\
\hline$C(7)$ & $78(4)$ & $62(3)$ & $55(3)$ & $9(2)$ & $8(2)$ & $12(3)$ \\
\hline$C(8)$ & $48(3)$ & $61(3)$ & $134(6)$ & $-24(3)$ & $-33(3)$ & $8(3)$ \\
\hline$C(9)$ & $49(3)$ & $60(3)$ & $67(3)$ & $-6(2)$ & $5(2)$ & $14(2)$ \\
\hline$C(10)$ & $32(2)$ & $54(3)$ & $61(3)$ & $-19(2)$ & $-5(2)$ & $5(2)$ \\
\hline$C(11)$ & $44(2)$ & $49(3)$ & $71(3)$ & $-17(2)$ & $-10(2)$ & $1(2)$ \\
\hline$C(12)$ & $55(3)$ & $71(4)$ & $132(6)$ & $-38(4)$ & $-49(3)$ & $23(3)$ \\
\hline$C(13)$ & $49(3)$ & $109(5)$ & $103(5)$ & $-44(4)$ & $33(3)$ & $-34(3)$ \\
\hline$C(14)$ & $29(2)$ & $49(3)$ & $52(3)$ & $-3(2)$ & $9(2)$ & $-6(2)$ \\
\hline$C(15)$ & $36(2)$ & $56(3)$ & $57(3)$ & $8(2)$ & $7(2)$ & $-6(2)$ \\
\hline$C(16)$ & $32(2)$ & $48(2)$ & $43(3)$ & $-2(2)$ & $1(2)$ & $-3(2)$ \\
\hline$C(17)$ & $54(3)$ & $52(3)$ & $61(3)$ & $-5(2)$ & $-10(2)$ & $-9(2)$ \\
\hline $\operatorname{Si}(4)$ & $29(1)$ & $44(1)$ & $43(1)$ & $0(1)$ & $5(1)$ & $1(1)$ \\
\hline$C(18)$ & $34(2)$ & $58(3)$ & $55(3)$ & $3(2)$ & $4(2)$ & $-4(2)$ \\
\hline$C(19)$ & $35(2)$ & $50(3)$ & $43(3)$ & $7(2)$ & $3(2)$ & $3(2)$ \\
\hline$C(20)$ & $43(2)$ & $75(3)$ & $52(3)$ & $6(2)$ & $-8(2)$ & $1(2)$ \\
\hline$C(21)$ & $50(3)$ & $60(3)$ & $58(3)$ & $15(2)$ & $8(2)$ & $-2(2)$ \\
\hline$C(22)$ & $45(2)$ & $44(3)$ & $55(3)$ & $5(2)$ & $5(2)$ & $9(2)$ \\
\hline$C(23)$ & $41(2)$ & $49(3)$ & $55(3)$ & $-1(2)$ & $15(2)$ & $6(2)$ \\
\hline
\end{tabular}




$\begin{array}{lllllll}\mathrm{C}(24) & 64(3) & 77(4) & 53(3) & -7(2) & 25(2) & -3(3) \\ \mathrm{C}(25) & 54(3) & 60(3) & 60(3) & -21(2) & 14(2) & -2(2) \\ \mathrm{C}(26) & 49(3) & 58(3) & 74(4) & -2(2) & 25(2) & 7(2) \\ \mathrm{C}(27) & 45(2) & 50(3) & 57(3) & -4(2) & -7(2) & 9(2) \\ \mathrm{C}(28) & 47(2) & 43(3) & 67(3) & -5(2) & 3(2) & 7(2) \\ \mathrm{C}(29) & 68(3) & 49(3) & 73(4) & 7(2) & -7(3) & 11(2) \\ \mathrm{C}(30) & 94(5) & 80(5) & 97(5) & 23(4) & -30(4) & -14(4) \\ \mathrm{Si}(5) & 25(1) & 40(1) & 38(1) & 0(1) & 4(1) & 0(1) \\ \mathrm{C}(31) & 29(2) & 45(2) & 42(2) & -2(2) & 6(2) & 1(2) \\ \mathrm{C}(32) & 28(2) & 35(2) & 45(2) & -1(2) & 5(2) & 1(2) \\ \mathrm{Si}(6) & 27(1) & 39(1) & 36(1) & 1(1) & 4(1) & 1(1) \\ \mathrm{C}(33) & 32(2) & 53(3) & 42(3) & 1(2) & 5(2) & 5(2) \\ \mathrm{C}(34) & 29(2) & 40(2) & 44(2) & 1(2) & 5(2) & 2(2) \\ \mathrm{Si}(7) & 30(1) & 42(1) & 39(1) & 3(1) & 4(1) & 0(1) \\ \mathrm{C}(35) & 44(2) & 54(3) & 46(3) & 4(2) & 8(2) & -1(2) \\ \mathrm{C}(36) & 35(2) & 38(2) & 42(2) & 2(2) & -3(2) & 2(2) \\ \mathrm{C}(37) & 35(2) & 50(3) & 61(3) & 3(2) & -2(2) & 4(2) \\ \mathrm{C}(38) & 35(2) & 58(3) & 62(3) & -9(2) & 1(2) & 12(2) \\ \mathrm{C}(39) & 46(3) & 57(3) & 57(3) & 15(2) & -2(2) & 3(2) \\ \mathrm{C}(40) & 33(2) & 41(2) & 46(3) & 4(2) & 4(2) & -3(2) \\ \mathrm{C}(41) & 37(2) & 52(3) & 50(3) & -2(2) & -1(2) & -5(2) \\ \mathrm{C}(42) & 42(2) & 57(3) & 70(3) & 12(2) & -1(2) & -11(2) \\ \mathrm{C}(43) & 36(2) & 59(3) & 58(3) & 0(2) & 11(2) & -1(2) \\ \mathrm{C}(44) & 32(2) & 55(3) & 38(2) & -7(2) & 1(2) & -4(2) \\ \mathrm{C}(45) & 47(3) & 68(3) & 48(3) & -16(2) & 8(2) & -9(2) \\ \mathrm{C}(46) & 30(2) & 39(2) & 45(2) & -2(2) & 4(2) & -4(2) \\ \mathrm{C}(47) & 43(2) & 52(3) & 51(3) & 5(2) & 3(2) & -9(2) \\ \mathrm{Si}(8) & 27(1) & 40(1) & 39(1) & 0(1) & 3(1) & 1(1) \\ \mathrm{C}(48) & 41(2) & 47(3) & 45(3) & 4(2) & 3(2) & -1(2) \\ \mathrm{C}(49) & 31(2) & 47(2) & 44(3) & -1(2) & 0(2) & 0(2) \\ \mathrm{C}(50) & 45(2) & 53(3) & 46(3) & -7(2) & -2(2) & -5(2) \\ \mathrm{C}(51) & 50(3) & 44(3) & 57(3) & 6(2) & 3(2) & -4(2) \\ \mathrm{C}(52) & 32(2) & 65(3) & 49(3) & 0(2) & 4(2) & -3(2)\end{array}$




$\begin{array}{lllllll}\mathrm{C}(53) & 29(2) & 48(2) & 46(3) & -4(2) & 3(2) & 1(2) \\ \mathrm{C}(54) & 35(2) & 65(3) & 55(3) & -2(2) & -1(2) & 12(2) \\ \mathrm{C}(55) & 38(2) & 66(3) & 57(3) & -15(2) & 10(2) & 4(2) \\ \mathrm{C}(56) & 33(2) & 58(3) & 63(3) & -8(2) & 10(2) & -2(2) \\ \mathrm{C}(57) & 49(3) & 68(3) & 47(3) & 14(2) & 13(2) & 21(2) \\ \mathrm{C}(58) & 76(4) & 71(4) & 91(5) & 0(3) & -21(3) & 10(3) \\ \mathrm{C}(59) & 26(2) & 63(3) & 51(3) & 5(2) & 9(2) & 9(2) \\ \mathrm{C}(60) & 33(2) & 53(3) & 47(3) & -6(2) & 9(2) & 1(2)\end{array}$


Table 5. Hydrogen coordinates $\left(\times 10^{4}\right)$ and isotropic displacement parameters $\left(\AA^{2} \times 10^{3}\right)$ for 1,4-disila(Dewer-benzene).

\begin{tabular}{|c|c|c|c|c|}
\hline & $x$ & $\mathrm{y}$ & $\mathrm{z}$ & $\mathrm{U}(\mathrm{eq})$ \\
\hline $\mathrm{H}(1)$ & 5324 & 1768 & 2338 & 96 \\
\hline $\mathrm{H}(2)$ & 4455 & 2325 & 2233 & 96 \\
\hline $\mathrm{H}(3)$ & 5063 & 3217 & 2451 & 96 \\
\hline $\mathrm{H}(4)$ & 5169 & 5258 & 1356 & 97 \\
\hline $\mathrm{H}(5)$ & 5306 & 6288 & 1641 & 97 \\
\hline $\mathrm{H}(6)$ & 4475 & 6260 & 1433 & 97 \\
\hline $\mathrm{H}(7)$ & 3706 & 3171 & 1710 & 125 \\
\hline $\mathrm{H}(8)$ & 4103 & 3401 & 1390 & 125 \\
\hline $\mathrm{H}(9)$ & 3480 & 4466 & 1501 & 125 \\
\hline $\mathrm{H}(10)$ & 3869 & 6116 & 1928 & 88 \\
\hline $\mathrm{H}(11)$ & 4685 & 5919 & 2141 & 88 \\
\hline $\mathrm{H}(12)$ & 3986 & 4847 & 2154 & 88 \\
\hline $\mathrm{H}(13)$ & 6923 & 5717 & 2317 & 83 \\
\hline $\mathrm{H}(14)$ & 6142 & 5091 & 2443 & 83 \\
\hline $\mathrm{H}(15)$ & 6067 & 6078 & 2149 & 83 \\
\hline $\mathrm{H}(16)$ & 7048 & 2295 & 2076 & 135 \\
\hline $\mathrm{H}(17)$ & 6697 & 2731 & 2394 & 135 \\
\hline $\mathrm{H}(18)$ & 7482 & 3435 & 2290 & 135 \\
\hline $\mathrm{H}(19)$ & 6551 & 5309 & 1634 & 128 \\
\hline $\mathrm{H}(20)$ & 6900 & 3830 & 1603 & 128 \\
\hline $\mathrm{H}(21)$ & 7393 & 4929 & 1810 & 128 \\
\hline $\mathrm{H}(22)$ & 3534 & 917 & 1453 & 51 \\
\hline $\mathrm{H}(23)$ & 3639 & -486 & 1281 & 51 \\
\hline $\mathrm{H}(24)$ & 4379 & -1334 & 1735 & 75 \\
\hline $\mathrm{H}(25)$ & 3528 & -839 & 1822 & 75 \\
\hline$H(26)$ & 4294 & 74 & 1906 & 75 \\
\hline $\mathrm{H}(27)$ & 3891 & -136 & 770 & 49 \\
\hline $\mathrm{H}(28)$ & 4602 & 65 & 554 & 49 \\
\hline
\end{tabular}




\begin{tabular}{|c|c|c|c|c|}
\hline $\mathrm{H}(29)$ & 5160 & -2026 & 733 & 85 \\
\hline $\mathrm{H}(30)$ & 4278 & -2238 & 574 & 85 \\
\hline $\mathrm{H}(31)$ & 4440 & -2229 & 946 & 85 \\
\hline $\mathrm{H}(32)$ & 7963 & 3346 & 714 & 73 \\
\hline $\mathrm{H}(33)$ & 7895 & 2394 & 1009 & 73 \\
\hline $\mathrm{H}(34)$ & 7461 & 3819 & 992 & 73 \\
\hline $\mathrm{H}(35)$ & 4999 & 2420 & 361 & 86 \\
\hline $\mathrm{H}(36)$ & 5491 & 2834 & 75 & 86 \\
\hline $\mathrm{H}(37)$ & 4904 & 3891 & 214 & 86 \\
\hline $\mathrm{H}(38)$ & 6079 & 5481 & 255 & 84 \\
\hline $\mathrm{H}(39)$ & 6719 & 4393 & 170 & 84 \\
\hline $\mathrm{H}(40)$ & 6843 & 5196 & 492 & 84 \\
\hline $\mathrm{H}(41)$ & 6015 & 4589 & 945 & 72 \\
\hline $\mathrm{H}(42)$ & 5239 & 3688 & 871 & 72 \\
\hline $\mathrm{H}(43)$ & 5296 & 5128 & 711 & 72 \\
\hline $\mathrm{H}(44)$ & 7733 & 2351 & 131 & 95 \\
\hline $\mathrm{H}(45)$ & 6863 & 2249 & -47 & 95 \\
\hline $\mathrm{H}(46)$ & 7495 & 1081 & -84 & 95 \\
\hline $\mathrm{H}(47)$ & 6499 & -668 & 97 & 86 \\
\hline $\mathrm{H}(48)$ & 5874 & 521 & 124 & 86 \\
\hline $\mathrm{H}(49)$ & 6104 & -433 & 417 & 86 \\
\hline $\mathrm{H}(50)$ & 7550 & -327 & 688 & 89 \\
\hline $\mathrm{H}(51)$ & 8168 & 694 & 555 & 89 \\
\hline $\mathrm{H}(52)$ & 7849 & -562 & 350 & 89 \\
\hline $\mathrm{H}(53)$ & 7368 & -1793 & 1511 & 62 \\
\hline $\mathrm{H}(54)$ & 7680 & -997 & 1224 & 62 \\
\hline $\mathrm{H}(55)$ & 6353 & -2813 & 1168 & 79 \\
\hline $\mathrm{H}(56)$ & 7207 & -3142 & 1055 & 79 \\
\hline $\mathrm{H}(57)$ & 6662 & -2014 & 880 & 79 \\
\hline $\mathrm{H}(58)$ & 5958 & -659 & 2074 & 77 \\
\hline $\mathrm{H}(59)$ & 6383 & -1781 & 1885 & 77 \\
\hline $\mathrm{H}(60)$ & 7618 & -795 & 2049 & 139 \\
\hline $\mathrm{H}(61)$ & 7161 & -1343 & 2333 & 139 \\
\hline
\end{tabular}




\begin{tabular}{|c|c|c|c|c|}
\hline $\mathrm{H}(62)$ & 7165 & 227 & 2258 & 139 \\
\hline $\mathrm{H}(63)$ & 10126 & 1912 & 2409 & 72 \\
\hline $\mathrm{H}(64)$ & 9888 & 3391 & 2292 & 72 \\
\hline $\mathrm{H}(65)$ & 10794 & 3042 & 2399 & 72 \\
\hline $\mathrm{H}(66)$ & 12657 & 1658 & 2002 & 73 \\
\hline$H(67)$ & 12077 & 2715 & 2145 & 73 \\
\hline $\mathrm{H}(68)$ & 12198 & 2744 & 1780 & 73 \\
\hline $\mathrm{H}(69)$ & 12095 & 19 & 1597 & 77 \\
\hline $\mathrm{H}(70)$ & 11501 & 1051 & 1412 & 77 \\
\hline $\mathrm{H}(71)$ & 11164 & -293 & 1554 & 77 \\
\hline $\mathrm{H}(72)$ & 11060 & -525 & 2136 & 81 \\
\hline $\mathrm{H}(73)$ & 11447 & 591 & 2369 & 81 \\
\hline $\mathrm{H}(74)$ & 12004 & -351 & 2181 & 81 \\
\hline$H(75)$ & 9948 & 614 & 1274 & 70 \\
\hline $\mathrm{H}(76)$ & 9508 & 2035 & 1249 & 70 \\
\hline $\mathrm{H}(77)$ & 9000 & 685 & 1253 & 70 \\
\hline $\mathrm{H}(78)$ & 9549 & -228 & 2063 & 85 \\
\hline $\mathrm{H}(79)$ & 9909 & -806 & 1759 & 85 \\
\hline $\mathrm{H}(80)$ & 8969 & -666 & 1766 & 85 \\
\hline $\mathrm{H}(81)$ & 8286 & 1508 & 1690 & 76 \\
\hline $\mathrm{H}(82)$ & 8770 & 2887 & 1708 & 76 \\
\hline $\mathrm{H}(83)$ & 8746 & 1973 & 2012 & 76 \\
\hline $\mathrm{H}(84)$ & 12274 & 5367 & 2036 & 50 \\
\hline $\mathrm{H}(85)$ & 12137 & 6856 & 1906 & 50 \\
\hline $\mathrm{H}(86)$ & 10876 & 6919 & 2107 & 82 \\
\hline$H(87)$ & 11551 & 6599 & 2381 & 82 \\
\hline $\mathrm{H}(88)$ & 11001 & 5418 & 2233 & 82 \\
\hline $\mathrm{H}(89)$ & 12795 & 6497 & 1221 & 45 \\
\hline $\mathrm{H}(90)$ & 12396 & 7491 & 1453 & 45 \\
\hline $\mathrm{H}(91)$ & 11892 & 7265 & 798 & 73 \\
\hline $\mathrm{H}(92)$ & 12390 & 8497 & 951 & 73 \\
\hline $\mathrm{H}(93)$ & 11494 & 8261 & 1030 & 73 \\
\hline $\mathrm{H}(94)$ & 11413 & 6030 & 330 & 67 \\
\hline
\end{tabular}




\begin{tabular}{|c|c|c|c|c|}
\hline $\mathrm{H}(95)$ & 10611 & 5305 & 196 & 67 \\
\hline $\mathrm{H}(96)$ & 11435 & 4924 & 62 & 67 \\
\hline $\mathrm{H}(97)$ & 11188 & 1828 & 24 & 73 \\
\hline $\mathrm{H}(98)$ & 10447 & 2812 & -48 & 73 \\
\hline $\mathrm{H}(99)$ & 10303 & 1256 & 16 & 73 \\
\hline $\mathrm{H}(100)$ & 10435 & 335 & 567 & 75 \\
\hline $\mathrm{H}(101)$ & 10880 & 1200 & 842 & 75 \\
\hline $\mathrm{H}(102)$ & 11360 & 662 & 564 & 75 \\
\hline $\mathrm{H}(103)$ & 9616 & 3555 & 375 & 73 \\
\hline $\mathrm{H}(104)$ & 9740 & 2892 & 715 & 73 \\
\hline $\mathrm{H}(105)$ & 9402 & 2010 & 422 & 73 \\
\hline $\mathrm{H}(106)$ & 12419 & 1664 & 892 & 78 \\
\hline $\mathrm{H}(107)$ & 12562 & 3024 & 1085 & 78 \\
\hline $\mathrm{H}(108)$ & 13278 & 2347 & 924 & 78 \\
\hline $\mathrm{H}(109)$ & 13297 & 2423 & 327 & 80 \\
\hline $\mathrm{H}(110)$ & 12541 & 2994 & 119 & 80 \\
\hline $\mathrm{H}(111)$ & 12471 & 1637 & 313 & 80 \\
\hline $\mathrm{H}(112)$ & 12823 & 5228 & 792 & 77 \\
\hline $\mathrm{H}(113)$ & 12871 & 5182 & 422 & 77 \\
\hline $\mathrm{H}(114)$ & 13540 & 4464 & 650 & 77 \\
\hline $\mathrm{H}(115)$ & 9432 & 5930 & 613 & 65 \\
\hline $\mathrm{H}(116)$ & 8830 & 6247 & 870 & 65 \\
\hline $\mathrm{H}(117)$ & 9614 & 8123 & 1033 & 121 \\
\hline $\mathrm{H}(118)$ & 9265 & 8251 & 676 & 121 \\
\hline$H(119)$ & 10171 & 7826 & 760 & 121 \\
\hline $\mathrm{H}(120)$ & 8767 & 6509 & 1393 & 56 \\
\hline $\mathrm{H}(121)$ & 8538 & 5000 & 1470 & 56 \\
\hline $\mathrm{H}(122)$ & 9268 & 5201 & 1969 & 66 \\
\hline $\mathrm{H}(123)$ & 8568 & 6282 & 1928 & 66 \\
\hline $\mathrm{H}(124)$ & 9463 & 6727 & 1891 & 66 \\
\hline
\end{tabular}


Table 6. Crystal data and structure refinement for disilylketone.

Identification code

Empirical formula

Formula weight

Temperature

Wavelength

Crystal system

Space group

Unit cell dimensions

Volume

Z

Density (calculated)

Absorption coefficient

$\mathrm{F}(000)$

Crystal size

Theta range for data collection

Index ranges

Reflections collected

Independent reflections

Completeness to theta $=27.98^{\circ}$

Max. and min. transmission

Refinement method

Data / restraints / parameters

Goodness-of-fit on $\mathrm{F}^{2}$

Final $\mathrm{R}$ indices [I $>2 \operatorname{sigma}(\mathrm{I})]$

$\mathrm{R}$ indices (all data)

Largest diff. peak and hole disilylketone

C31 H62 O Si 4

563.17

$120(2) \mathrm{K}$

$0.71070 \AA$

monoclinic

$P 2{ }_{1} / \mathrm{c}$

$a=8.8570(4) \AA$

$\alpha=90^{\circ}$.

$b=25.9730(9) \AA$

$\beta=104.6070(5)^{\circ}$.

$c=15.8440(6) \AA$

$\gamma=90^{\circ}$.

$3527.0(2) \AA^{3}$

4

$1.061 \mathrm{Mg} / \mathrm{m}^{3}$

$0.189 \mathrm{~mm}^{-1}$

1248

$0.40 \times 0.30 \times 0.25 \mathrm{~mm}^{3}$

2.38 to $27.98^{\circ}$.

$0<=\mathrm{h}<=11,0<=\mathrm{k}<=34,-20<=\mathrm{l}<=20$

8053

$8053[R($ int $)=0.032]$

$94.7 \%$

0.9542 and 0.9282

Full-matrix least-squares on $\mathrm{F}^{2}$

$8053 / 0 / 427$

1.029

$R_{1}=0.0436, \mathrm{w} R_{2}=0.1173$

$R_{1}=0.0569, \mathrm{w} R_{2}=0.1254$

0.378 and -0.595 e. $\AA^{-3}$ 

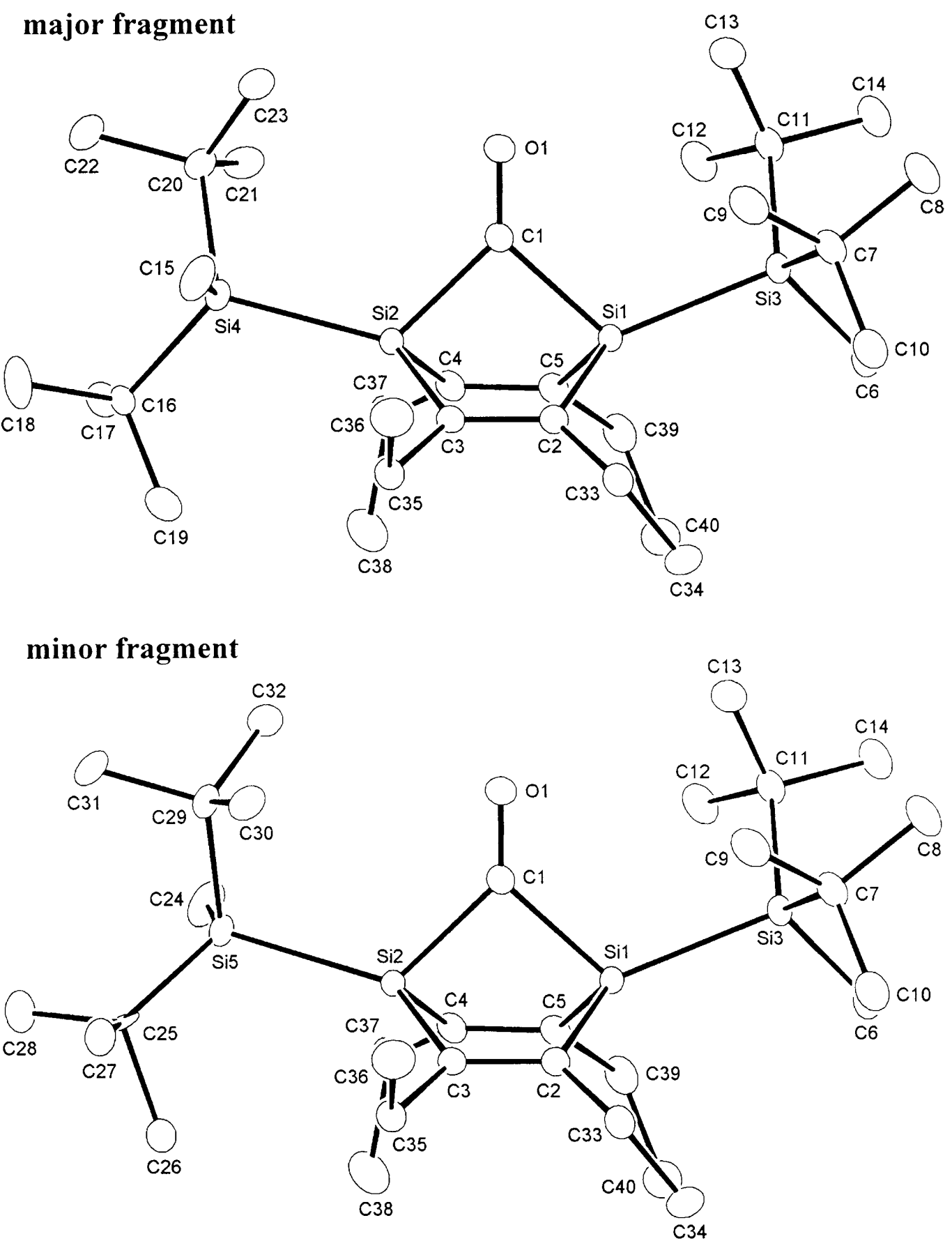

Figure 2. ORTEP drawing of 1,4-bis(di-tert-butylmethylsilyl)-2,3,5,6-tetraethyl-1,4-disilabicyclo[2.2.1] hepta-2,5-dien-7-one (4) (30\% probability) with nonhydrogen atoms. 
Table 7. Atomic coordinates ( $\left.\times 10^{4}\right)$ and equivalent isotropic displacement parameters $\left(\AA^{2} \mathrm{x}\right.$ $10^{3}$ )

for disilylketone. $U(\mathrm{eq})$ is defined as one third of the trace of the orthogonalized Uij tensor.

\begin{tabular}{|c|c|c|c|c|}
\hline & $\mathrm{x}$ & $\mathrm{y}$ & z & $\mathrm{U}(\mathrm{eq})$ \\
\hline$C(1)$ & $6224(2)$ & $1066(1)$ & $1693(1)$ & $31(1)$ \\
\hline $\mathrm{O}(1)$ & $4907(2)$ & $913(1)$ & $1562(1)$ & $57(1)$ \\
\hline $\mathrm{Si}(1)$ & $8256(1)$ & $726(1)$ & $2155(1)$ & $22(1)$ \\
\hline$C(2)$ & $8831(2)$ & $884(1)$ & $1114(1)$ & $25(1)$ \\
\hline$C(3)$ & $8299(2)$ & $1352(1)$ & $779(1)$ & $25(1)$ \\
\hline $\operatorname{Si}(2)$ & $7191(1)$ & $1721(1)$ & $1459(1)$ & $25(1)$ \\
\hline$C(4)$ & $8473(2)$ & 1743(1) & $2607(1)$ & $27(1)$ \\
\hline$C(5)$ & $9030(2)$ & $1274(1)$ & 2921(1) & $27(1)$ \\
\hline $\operatorname{Si}(3)$ & $8145(1)$ & $-109(1)$ & $2775(1)$ & $24(1)$ \\
\hline$C(6)$ & $10056(2)$ & $-269(1)$ & $3570(1)$ & $37(1)$ \\
\hline$C(7)$ & $7739(2)$ & $-630(1)$ & $1886(1)$ & $31(1)$ \\
\hline $\mathrm{C}(8)$ & $7161(3)$ & $-1134(1)$ & $2218(1)$ & $41(1)$ \\
\hline$C(9)$ & $6525(2)$ & $-448(1)$ & $1069(1)$ & $41(1)$ \\
\hline$C(10)$ & $9255(2)$ & $-763(1)$ & $1640(1)$ & $41(1)$ \\
\hline$C(11)$ & $6578(2)$ & $-72(1)$ & $3430(1)$ & $31(1)$ \\
\hline$C(12)$ & $6721(3)$ & $441(1)$ & $3927(1)$ & $43(1)$ \\
\hline$C(13)$ & $4909(2)$ & $-110(1)$ & $2856(1)$ & $42(1)$ \\
\hline$C(14)$ & $6838(3)$ & $-504(1)$ & $4118(1)$ & $41(1)$ \\
\hline $\operatorname{Si}(4)$ & $5590(1)$ & $2412(1)$ & $747(1)$ & $24(1)$ \\
\hline$C(15)$ & $4849(3)$ & $2217(1)$ & $-427(1)$ & $42(1)$ \\
\hline$C(16)$ & $6720(7)$ & $3055(2)$ & $753(4)$ & $27(1)$ \\
\hline$C(17)$ & $6936(3)$ & $3361(1)$ & $1603(2)$ & $38(1)$ \\
\hline$C(18)$ & $5786(4)$ & $3401(1)$ & $6(2)$ & $55(1)$ \\
\hline$C(19)$ & $8309(6)$ & $2945(2)$ & $602(3)$ & $47(1)$ \\
\hline$C(20)$ & $3823(3)$ & $2444(1)$ & $1239(2)$ & $31(1)$ \\
\hline$C(21)$ & $4335(3)$ & $2453(1)$ & $2240(2)$ & $41(1)$ \\
\hline$C(22)$ & $2814(3)$ & $2920(1)$ & $916(2)$ & $42(1)$ \\
\hline
\end{tabular}




$\begin{array}{lcccc}\mathrm{C}(23) & 2806(3) & 1968(1) & 943(2) & 44(1) \\ \mathrm{Si}(5) & 5685(2) & 2513(1) & 1260(2) & 24(1) \\ \mathrm{C}(24) & 5633(11) & 2815(4) & 2337(5) & 42(2) \\ \mathrm{C}(25) & 6380(30) & 3002(10) & 595(15) & 30(4) \\ \mathrm{C}(26) & 8270(20) & 3035(6) & 953(11) & 49(4) \\ \mathrm{C}(27) & 6157(11) & 2906(3) & -360(6) & 41(2) \\ \mathrm{C}(28) & 5869(13) & 3555(3) & 740(7) & 46(2) \\ \mathrm{C}(29) & 3599(10) & 2297(3) & 747(6) & 29(2) \\ \mathrm{C}(30) & 3517(10) & 1905(3) & 6(6) & 38(2) \\ \mathrm{C}(31) & 2534(10) & 2760(4) & 363(7) & 40(2) \\ \mathrm{C}(32) & 2926(12) & 2041(4) & 1450(8) & 41(2) \\ \mathrm{C}(33) & 9799(2) & 529(1) & 702(1) & 30(1) \\ \mathrm{C}(34) & 11417(2) & 434(1) & 1300(1) & 41(1) \\ \mathrm{C}(35) & 8528(2) & 1540(1) & -90(1) & 31(1) \\ \mathrm{C}(36) & 7451(2) & 1265(1) & -872(1) & 48(1) \\ \mathrm{C}(37) & 8849(2) & 2239(1) & 3128(1) & 38(1) \\ \mathrm{C}(38) & 10375(3) & 2488(1) & 3062(1) & 50(1) \\ \mathrm{C}(39) & 10234(2) & 1191(1) & 3778(1) & 36(1) \\ \mathrm{C}(40) & 11828(2) & 1077(1) & 3620(1) & 46(1)\end{array}$


Table 8. Bond lengths $[\AA]$ and angles $\left[{ }^{\circ}\right]$ for disilylketone.

\begin{tabular}{|c|c|c|c|}
\hline $\mathrm{C}(1)-\mathrm{O}(1)$ & $1.199(2)$ & $C(20)-C(23)$ & $1.534(3)$ \\
\hline$C(1)-\operatorname{Si}(1)$ & $1.9730(17)$ & $C(20)-C(22)$ & $1.535(3)$ \\
\hline$C(1)-\operatorname{Si}(2)$ & $1.9807(16)$ & $C(20)-C(21)$ & $1.535(3)$ \\
\hline $\operatorname{Si}(1)-C(5)$ & $1.8847(15)$ & $\mathrm{Si}(5)-\mathrm{C}(25)$ & $1.85(3)$ \\
\hline $\operatorname{Si}(1)-C(2)$ & $1.8906(15)$ & $\operatorname{Si}(5)-C(24)$ & $1.890(8)$ \\
\hline $\operatorname{Si}(1)-\operatorname{Si}(3)$ & $2.3919(5)$ & $\operatorname{Si}(5)-C(29)$ & $1.905(9)$ \\
\hline$C(2)-C(3)$ & $1.362(2)$ & $C(25)-C(27)$ & $1.50(3)$ \\
\hline$C(2)-C(33)$ & $1.513(2)$ & $C(25)-C(28)$ & $1.54(3)$ \\
\hline$C(3)-C(35)$ & $1.523(2)$ & $\mathrm{C}(25)-\mathrm{C}(26)$ & $1.63(3)$ \\
\hline$C(3)-\operatorname{Si}(2)$ & $1.8902(16)$ & $\mathrm{C}(29)-\mathrm{C}(30)$ & $1.542(12)$ \\
\hline $\operatorname{Si}(2)-C(4)$ & $1.8873(17)$ & $C(29)-C(32)$ & $1.541(12)$ \\
\hline $\operatorname{Si}(2)-\operatorname{Si}(4)$ & $2.3874(6)$ & $\mathrm{C}(29)-\mathrm{C}(31)$ & $1.554(11)$ \\
\hline $\operatorname{Si}(2)-\operatorname{Si}(5)$ & $2.428(2)$ & $C(33)-C(34)$ & $1.526(3)$ \\
\hline$C(4)-C(5)$ & $1.360(2)$ & $C(35)-C(36)$ & $1.537(3)$ \\
\hline$C(4)-C(37)$ & $1.521(2)$ & $C(37)-C(38)$ & $1.525(3)$ \\
\hline$C(5)-C(39)$ & $1.515(2)$ & $C(39)-C(40)$ & $1.524(3)$ \\
\hline $\operatorname{Si}(3)-C(6)$ & $1.8832(18)$ & & \\
\hline $\operatorname{Si}(3)-C(7)$ & $1.9201(16)$ & $\mathrm{O}(1)-\mathrm{C}(1)-\mathrm{Si}(1)$ & $132.22(13)$ \\
\hline $\mathrm{Si}(3)-\mathrm{C}(11)$ & $1.9335(17)$ & $\mathrm{O}(1)-\mathrm{C}(1)-\mathrm{Si}(2)$ & $134.39(13)$ \\
\hline$C(7)-C(10)$ & $1.530(2)$ & $\mathrm{Si}(1)-\mathrm{C}(1)-\mathrm{Si}(2)$ & $93.26(7)$ \\
\hline$C(7)-C(9)$ & $1.534(2)$ & $C(5)-S i(1)-C(2)$ & $105.33(7)$ \\
\hline$C(7)-C(8)$ & $1.546(2)$ & $C(5)-S i(1)-C(1)$ & $92.88(7)$ \\
\hline$C(11)-C(13)$ & $1.531(3)$ & $C(2)-\operatorname{Si}(1)-C(1)$ & $90.00(7)$ \\
\hline$C(11)-C(12)$ & $1.536(2)$ & $\mathrm{C}(5)-\mathrm{Si}(1)-\mathrm{Si}(3)$ & $117.79(5)$ \\
\hline$C(11)-C(14)$ & $1.542(2)$ & $\mathrm{C}(2)-\mathrm{Si}(1)-\mathrm{Si}(3)$ & $127.07(5)$ \\
\hline $\operatorname{Si}(4)-C(15)$ & $1.879(2)$ & $\mathrm{C}(1)-\mathrm{Si}(1)-\mathrm{Si}(3)$ & $115.48(5)$ \\
\hline $\mathrm{Si}(4)-\mathrm{C}(20)$ & $1.919(2)$ & $\mathrm{C}(3)-\mathrm{C}(2)-\mathrm{C}(33)$ & $123.61(14)$ \\
\hline $\operatorname{Si}(4)-C(16)$ & $1.945(5)$ & $\mathrm{C}(3)-\mathrm{C}(2)-\mathrm{Si}(1)$ & $112.94(11)$ \\
\hline$C(16)-C(19)$ & $1.513(8)$ & $\mathrm{C}(33)-\mathrm{C}(2)-\mathrm{Si}(1)$ & $123.44(11)$ \\
\hline$C(16)-C(17)$ & $1.533(7)$ & $C(2)-C(3)-C(35)$ & $121.82(14)$ \\
\hline$C(16)-C(18)$ & $1.550(5)$ & $\mathrm{C}(2)-\mathrm{C}(3)-\mathrm{Si}(2)$ & $114.21(11)$ \\
\hline
\end{tabular}




\begin{tabular}{|c|c|c|c|}
\hline$C(35)-C(3)-\operatorname{Si}(2)$ & $123.92(11)$ & $\mathrm{C}(12)-\mathrm{C}(11)-\mathrm{Si}(3)$ & $109.93(11)$ \\
\hline$C(4)-\operatorname{Si}(2)-C(3)$ & $106.98(7)$ & $\mathrm{C}(14)-\mathrm{C}(11)-\mathrm{Si}(3)$ & $110.08(12)$ \\
\hline $\mathrm{C}(4)-\mathrm{Si}(2)-\mathrm{C}(1)$ & $91.67(7)$ & $C(15)-S i(4)-C(20)$ & $107.28(12)$ \\
\hline$C(3)-S i(2)-C(1)$ & $88.97(7)$ & $C(15)-\operatorname{Si}(4)-C(16)$ & $106.73(19)$ \\
\hline$C(4)-\operatorname{Si}(2)-\operatorname{Si}(4)$ & $125.03(5)$ & $\mathrm{C}(20)-\mathrm{Si}(4)-\mathrm{C}(16)$ & $115.6(2)$ \\
\hline$C(3)-\operatorname{Si}(2)-\operatorname{Si}(4)$ & $116.36(5)$ & $\mathrm{C}(15)-\mathrm{Si}(4)-\operatorname{Si}(2)$ & $106.39(8)$ \\
\hline$C(1)-\operatorname{Si}(2)-\operatorname{Si}(4)$ & $120.06(5)$ & $\mathrm{C}(20)-\mathrm{Si}(4)-\operatorname{Si}(2)$ & $106.78(7)$ \\
\hline$C(4)-\operatorname{Si}(2)-\operatorname{Si}(5)$ & $106.04(8)$ & $C(16)-\operatorname{Si}(4)-\operatorname{Si}(2)$ & $113.56(17)$ \\
\hline$C(3)-\operatorname{Si}(2)-\operatorname{Si}(5)$ & $134.66(8)$ & $\mathrm{C}(19)-\mathrm{C}(16)-\mathrm{C}(17)$ & $108.8(4)$ \\
\hline$C(1)-\operatorname{Si}(2)-\operatorname{Si}(5)$ & $120.15(7)$ & $C(19)-C(16)-C(18)$ & $109.1(4)$ \\
\hline $\operatorname{Si}(4)-\operatorname{Si}(2)-\operatorname{Si}(5)$ & $20.04(6)$ & $\mathrm{C}(17)-\mathrm{C}(16)-\mathrm{C}(18)$ & 107.1(4) \\
\hline $\mathrm{C}(5)-\mathrm{C}(4)-\mathrm{C}(37)$ & $123.42(15)$ & $C(19)-C(16)-S i(4)$ & $109.6(4)$ \\
\hline $\mathrm{C}(5)-\mathrm{C}(4)-\mathrm{Si}(2)$ & $113.59(11)$ & $C(17)-C(16)-S i(4)$ & $113.5(3)$ \\
\hline$C(37)-C(4)-\operatorname{Si}(2)$ & $122.99(12)$ & $C(18)-C(16)-S i(4)$ & $108.6(3)$ \\
\hline$C(4)-C(5)-C(39)$ & $124.32(14)$ & $C(23)-C(20)-C(22)$ & $107.48(19)$ \\
\hline$C(4)-C(5)-S i(1)$ & $113.67(12)$ & $C(23)-C(20)-C(21)$ & $109.0(2)$ \\
\hline$C(39)-C(5)-S i(1)$ & $121.91(11)$ & $C(22)-C(20)-C(21)$ & $109.2(2)$ \\
\hline$C(6)-S i(3)-C(7)$ & $107.77(8)$ & $C(23)-C(20)-S i(4)$ & $108.45(18)$ \\
\hline$C(6)-\operatorname{Si}(3)-C(11)$ & $107.36(8)$ & $\mathrm{C}(22)-\mathrm{C}(20)-\mathrm{Si}(4)$ & $111.45(16)$ \\
\hline$C(7)-\operatorname{Si}(3)-C(11)$ & $113.68(7)$ & $C(21)-C(20)-S i(4)$ & $111.21(15)$ \\
\hline$C(6)-\operatorname{Si}(3)-\operatorname{Si}(1)$ & $110.58(6)$ & $\mathrm{C}(25)-\mathrm{Si}(5)-\mathrm{C}(24)$ & $108.4(9)$ \\
\hline$C(7)-S i(3)-S i(1)$ & $110.89(5)$ & $C(25)-\operatorname{Si}(5)-C(29)$ & $112.5(6)$ \\
\hline $\mathrm{C}(11)-\mathrm{Si}(3)-\mathrm{Si}(1)$ & $106.52(5)$ & $C(24)-\operatorname{Si}(5)-C(29)$ & $105.4(4)$ \\
\hline$C(10)-C(7)-C(9)$ & $109.14(15)$ & $C(25)-\operatorname{Si}(5)-\operatorname{Si}(2)$ & $114.0(9)$ \\
\hline$C(10)-C(7)-C(8)$ & $106.38(14)$ & $\mathrm{C}(24)-\mathrm{Si}(5)-\mathrm{Si}(2)$ & $111.9(3)$ \\
\hline$C(9)-C(7)-C(8)$ & $109.01(15)$ & $C(29)-\operatorname{Si}(5)-\operatorname{Si}(2)$ & $104.4(2)$ \\
\hline$C(10)-C(7)-S i(3)$ & $109.46(12)$ & $\mathrm{C}(27)-\mathrm{C}(25)-\mathrm{C}(28)$ & $109.8(16)$ \\
\hline$C(9)-C(7)-\operatorname{Si}(3)$ & $111.37(12)$ & $C(27)-C(25)-C(26)$ & $102.7(18)$ \\
\hline$C(8)-C(7)-S i(3)$ & $111.34(11)$ & $C(28)-C(25)-C(26)$ & $102.2(13)$ \\
\hline$C(13)-C(11)-C(12)$ & $107.71(15)$ & $C(27)-C(25)-S i(5)$ & $118.9(13)$ \\
\hline$C(13)-C(11)-C(14)$ & $108.79(14)$ & $C(28)-C(25)-\operatorname{Si}(5)$ & $113.9(17)$ \\
\hline$C(12)-C(11)-C(14)$ & $106.89(14)$ & $\mathrm{C}(26)-\mathrm{C}(25)-\mathrm{Si}(5)$ & $107.2(14)$ \\
\hline$C(13)-C(11)-S i(3)$ & $113.22(12)$ & $\mathrm{C}(30)-\mathrm{C}(29)-\mathrm{C}(32)$ & $108.2(8)$ \\
\hline
\end{tabular}




$\begin{array}{llll}\mathrm{C}(30)-\mathrm{C}(29)-\mathrm{C}(31) & 107.7(7) & \mathrm{C}(2)-\mathrm{C}(33)-\mathrm{C}(34) & 112.21(13) \\ \mathrm{C}(32)-\mathrm{C}(29)-\mathrm{C}(31) & 108.0(7) & \mathrm{C}(3)-\mathrm{C}(35)-\mathrm{C}(36) & 112.47(14) \\ \mathrm{C}(30)-\mathrm{C}(29)-\mathrm{Si}(5) & 111.9(6) & \mathrm{C}(4)-\mathrm{C}(37)-\mathrm{C}(38) & 113.49(15) \\ \mathrm{C}(32)-\mathrm{C}(29)-\mathrm{Si}(5) & 109.2(7) & \mathrm{C}(5)-\mathrm{C}(39)-\mathrm{C}(40) & 110.65(14) \\ \mathrm{C}(31)-\mathrm{C}(29)-\mathrm{Si}(5) & 111.6(6) & & \end{array}$

Symmetry transformations used to generate equivalent atoms: 
Table 9. Anisotropic displacement parameters $\left(\AA^{2} \times 10^{3}\right)$ for disilylketone. The anisotropic displacement factor exponent takes the form: $-2 \pi^{2}\left[h^{2} a^{* 2} U^{11}+\ldots+2 h k a^{*} b^{*} U^{12}\right]$

\begin{tabular}{|c|c|c|c|c|c|c|}
\hline & $\mathrm{U}^{11}$ & $\mathrm{U}^{22}$ & $\mathrm{U}^{33}$ & $U^{23}$ & $\mathrm{U}^{13}$ & $\mathrm{U}^{12}$ \\
\hline$C(1)$ & $26(1)$ & $26(1)$ & $41(1)$ & $8(1)$ & $8(1)$ & $0(1)$ \\
\hline$O(1)$ & $22(1)$ & $42(1)$ & $102(1)$ & $31(1)$ & $7(1)$ & $-3(1)$ \\
\hline $\operatorname{Si}(1)$ & $22(1)$ & $19(1)$ & $24(1)$ & $2(1)$ & $3(1)$ & $0(1)$ \\
\hline$C(2)$ & $23(1)$ & $25(1)$ & $26(1)$ & $0(1)$ & $3(1)$ & $-2(1)$ \\
\hline$C(3)$ & $22(1)$ & $26(1)$ & $26(1)$ & $3(1)$ & $3(1)$ & $-2(1)$ \\
\hline $\operatorname{Si}(2)$ & $21(1)$ & $21(1)$ & $35(1)$ & $6(1)$ & $7(1)$ & $2(1)$ \\
\hline$C(4)$ & $33(1)$ & $22(1)$ & $31(1)$ & $-1(1)$ & $14(1)$ & $-2(1)$ \\
\hline$C(5)$ & $29(1)$ & $25(1)$ & $26(1)$ & $-1(1)$ & $7(1)$ & $-3(1)$ \\
\hline $\operatorname{Si}(3)$ & $27(1)$ & $19(1)$ & $25(1)$ & $2(1)$ & $4(1)$ & $0(1)$ \\
\hline$C(6)$ & $39(1)$ & $30(1)$ & $38(1)$ & $5(1)$ & $0(1)$ & $2(1)$ \\
\hline$C(7)$ & $41(1)$ & $23(1)$ & $30(1)$ & $-2(1)$ & $10(1)$ & $-6(1)$ \\
\hline$C(8)$ & $61(1)$ & $24(1)$ & $41(1)$ & $-4(1)$ & $18(1)$ & $-10(1)$ \\
\hline$C(9)$ & $49(1)$ & $41(1)$ & $29(1)$ & $-1(1)$ & $4(1)$ & $-14(1)$ \\
\hline$C(10)$ & $55(1)$ & $27(1)$ & $47(1)$ & $-4(1)$ & $25(1)$ & $0(1)$ \\
\hline$C(11)$ & $40(1)$ & $23(1)$ & $33(1)$ & $4(1)$ & $14(1)$ & $0(1)$ \\
\hline$C(12)$ & $63(1)$ & $29(1)$ & $46(1)$ & $-3(1)$ & $32(1)$ & $-3(1)$ \\
\hline$C(13)$ & $35(1)$ & $41(1)$ & $54(1)$ & $9(1)$ & $17(1)$ & $-1(1)$ \\
\hline$C(14)$ & $58(1)$ & $32(1)$ & $36(1)$ & $7(1)$ & $19(1)$ & $-2(1)$ \\
\hline $\operatorname{Si}(4)$ & $25(1)$ & $22(1)$ & $23(1)$ & $1(1)$ & $4(1)$ & $5(1)$ \\
\hline$C(15)$ & $44(1)$ & $48(1)$ & $29(1)$ & $-4(1)$ & $-3(1)$ & $18(1)$ \\
\hline$C(16)$ & $27(3)$ & $21(1)$ & $31(2)$ & $8(2)$ & $6(2)$ & $0(2)$ \\
\hline$C(17)$ & $41(1)$ & $25(1)$ & $50(1)$ & $-3(1)$ & $14(1)$ & $-3(1)$ \\
\hline$C(18)$ & $82(2)$ & $34(1)$ & $47(2)$ & $18(1)$ & $12(1)$ & $12(1)$ \\
\hline$C(19)$ & $50(2)$ & $35(2)$ & $68(3)$ & $7(2)$ & $37(2)$ & $0(1)$ \\
\hline $\mathrm{C}(20)$ & $25(1)$ & $30(1)$ & $37(1)$ & $-2(1)$ & $7(1)$ & $4(1)$ \\
\hline $\mathrm{C}(21)$ & $33(1)$ & $56(1)$ & $38(1)$ & $0(1)$ & $16(1)$ & $2(1)$ \\
\hline $\mathrm{C}(22)$ & $30(1)$ & $36(1)$ & $58(2)$ & $-4(1)$ & $9(1)$ & $9(1)$ \\
\hline$C(23)$ & $26(1)$ & $37(1)$ & $69(2)$ & $-2(1)$ & $9(1)$ & $0(1)$ \\
\hline
\end{tabular}




$\begin{array}{lcccccc}\mathrm{Si}(5) & 27(1) & 21(1) & 23(1) & 0(1) & 6(1) & 7(1) \\ \mathrm{C}(24) & 45(5) & 43(5) & 31(4) & -15(3) & -3(3) & 16(4) \\ \mathrm{C}(25) & 3(6) & 38(8) & 42(8) & -9(5) & -6(5) & 2(5) \\ \mathrm{C}(26) & 38(6) & 27(6) & 86(12) & 14(7) & 23(8) & 6(4) \\ \mathrm{C}(27) & 46(5) & 41(4) & 42(5) & 17(4) & 23(4) & 14(4) \\ \mathrm{C}(28) & 54(6) & 30(4) & 57(6) & 9(4) & 16(5) & 8(4) \\ \mathrm{C}(29) & 31(4) & 23(3) & 33(4) & -1(3) & 10(3) & 10(3) \\ \mathrm{C}(30) & 30(5) & 41(4) & 40(4) & -7(4) & 4(3) & 4(4) \\ \mathrm{C}(31) & 27(5) & 43(5) & 47(5) & 3(4) & 6(4) & 14(4) \\ \mathrm{C}(32) & 36(5) & 44(5) & 52(6) & 9(5) & 26(5) & 7(4) \\ \mathrm{C}(33) & 37(1) & 25(1) & 31(1) & -1(1) & 13(1) & 0(1) \\ \mathrm{C}(34) & 27(1) & 36(1) & 61(1) & -9(1) & 14(1) & 0(1) \\ \mathrm{C}(35) & 31(1) & 34(1) & 29(1) & 9(1) & 6(1) & 0(1) \\ \mathrm{C}(36) & 43(1) & 67(1) & 30(1) & 7(1) & 2(1) & -4(1) \\ \mathrm{C}(37) & 60(1) & 25(1) & 34(1) & -5(1) & 19(1) & -5(1) \\ \mathrm{C}(38) & 59(1) & 37(1) & 48(1) & -6(1) & 4(1) & -18(1) \\ \mathrm{C}(39) & 45(1) & 32(1) & 25(1) & 1(1) & 1(1) & -8(1) \\ \mathrm{C}(40) & 35(1) & 53(1) & 41(1) & 9(1) & -8(1) & -7(1)\end{array}$


Table 10. Hydrogen coordinates ( $\left.x 10^{4}\right)$ and isotropic displacement parameters $\left(\AA^{2} \times 10^{3}\right)$ for disilylketone.

\begin{tabular}{|c|c|c|c|c|}
\hline & $\mathrm{x}$ & y & z & $\mathrm{U}(\mathrm{eq})$ \\
\hline $\mathrm{H}(1)$ & 10041 & -628 & 3756 & 56 \\
\hline $\mathrm{H}(2)$ & 10210 & -42 & 4079 & 56 \\
\hline $\mathrm{H}(3)$ & 10911 & -219 & 3287 & 56 \\
\hline $\mathrm{H}(4)$ & 6136 & -1076 & 2331 & 61 \\
\hline $\mathrm{H}(5)$ & 7903 & -1242 & 2758 & 61 \\
\hline $\mathrm{H}(6)$ & 7077 & -1403 & 1776 & 61 \\
\hline $\mathrm{H}(7)$ & 6882 & -128 & 856 & 61 \\
\hline $\mathrm{H}(8)$ & 5527 & -387 & 1214 & 61 \\
\hline $\mathrm{H}(9)$ & 6389 & -713 & 615 & 61 \\
\hline $\mathrm{H}(10)$ & 9034 & -1010 & 1156 & 61 \\
\hline $\mathrm{H}(11)$ & 9995 & -915 & 2144 & 61 \\
\hline $\mathrm{H}(12)$ & 9706 & -450 & 1460 & 61 \\
\hline $\mathrm{H}(13)$ & 6530 & 727 & 3510 & 64 \\
\hline $\mathrm{H}(14)$ & 7772 & 472 & 4312 & 64 \\
\hline$H(15)$ & 5951 & 451 & 4275 & 64 \\
\hline $\mathrm{H}(16)$ & 4169 & -63 & 3216 & 63 \\
\hline $\mathrm{H}(17)$ & 4751 & -450 & 2578 & 63 \\
\hline $\mathrm{H}(18)$ & 4740 & 157 & 2405 & 63 \\
\hline $\mathrm{H}(19)$ & 6068 & -473 & 4464 & 61 \\
\hline $\mathrm{H}(20)$ & 7891 & -475 & 4503 & 61 \\
\hline $\mathrm{H}(21)$ & 6721 & -839 & 3825 & 61 \\
\hline $\mathrm{H}(22)$ & 5726 & 2195 & -699 & 64 \\
\hline $\mathrm{H}(23)$ & 4098 & 2473 & -735 & 64 \\
\hline $\mathrm{H}(24)$ & 4338 & 1880 & -459 & 64 \\
\hline$H(25)$ & 5913 & 3432 & 1709 & 57 \\
\hline $\mathrm{H}(26)$ & 7469 & 3686 & 1555 & 57 \\
\hline $\mathrm{H}(27)$ & 7564 & 3159 & 2089 & 57 \\
\hline
\end{tabular}




\begin{tabular}{|c|c|c|c|c|}
\hline $\mathrm{H}(28)$ & 5617 & 3217 & -549 & 83 \\
\hline$H(29)$ & 6375 & 3718 & -21 & 83 \\
\hline $\mathrm{H}(30)$ & 4776 & 3488 & 116 & 83 \\
\hline $\mathrm{H}(31)$ & 8185 & 2745 & 64 & 70 \\
\hline $\mathrm{H}(32)$ & 8934 & 2748 & 1095 & 70 \\
\hline $\mathrm{H}(33)$ & 8836 & 3270 & 548 & 70 \\
\hline $\mathrm{H}(34)$ & 4983 & 2150 & 2449 & 62 \\
\hline $\mathrm{H}(35)$ & 3411 & 2447 & 2474 & 62 \\
\hline $\mathrm{H}(36)$ & 4937 & 2766 & 2435 & 62 \\
\hline$H(37)$ & 3432 & 3232 & 1098 & 62 \\
\hline $\mathrm{H}(38)$ & 1908 & 2920 & 1166 & 62 \\
\hline $\mathrm{H}(39)$ & 2458 & 2911 & 279 & 62 \\
\hline $\mathrm{H}(40)$ & 3418 & 1656 & 1141 & 67 \\
\hline $\mathrm{H}(41)$ & 2449 & 1965 & 305 & 67 \\
\hline $\mathrm{H}(42)$ & 1901 & 1977 & 1193 & 67 \\
\hline $\mathrm{H}(43)$ & 6683 & 2930 & 2641 & 63 \\
\hline $\mathrm{H}(44)$ & 5266 & 2561 & 2698 & 63 \\
\hline $\mathrm{H}(45)$ & 4923 & 3111 & 2232 & 63 \\
\hline $\mathrm{H}(46)$ & 8545 & 3094 & 1584 & 73 \\
\hline $\mathrm{H}(47)$ & 8663 & 3320 & 663 & 73 \\
\hline $\mathrm{H}(48)$ & 8735 & 2711 & 826 & 73 \\
\hline $\mathrm{H}(49)$ & 5042 & 2917 & -654 & 61 \\
\hline $\mathrm{H}(50)$ & 6577 & 2566 & -445 & 61 \\
\hline $\mathrm{H}(51)$ & 6708 & 3171 & -605 & 61 \\
\hline $\mathrm{H}(52)$ & 4729 & 3570 & 617 & 70 \\
\hline$H(53)$ & 6231 & 3790 & 349 & 70 \\
\hline $\mathrm{H}(54)$ & 6325 & 3656 & 1347 & 70 \\
\hline $\mathrm{H}(55)$ & 3933 & 2062 & -451 & 57 \\
\hline $\mathrm{H}(56)$ & 2429 & 1804 & -240 & 57 \\
\hline $\mathrm{H}(57)$ & 4137 & 1600 & 237 & 57 \\
\hline $\mathrm{H}(58)$ & 2959 & 2935 & -74 & 59 \\
\hline $\mathrm{H}(59)$ & 2491 & 3001 & 832 & 59 \\
\hline $\mathrm{H}(60)$ & 1480 & 2635 & 89 & 59 \\
\hline
\end{tabular}




$\begin{array}{lrrrr}\mathrm{H}(61) & 3581 & 1747 & 1702 & 62 \\ \mathrm{H}(62) & 1863 & 1921 & 1185 & 62 \\ \mathrm{H}(63) & 2906 & 2291 & 1910 & 62 \\ \mathrm{H}(64) & 9909 & 684 & 149 & 36 \\ \mathrm{H}(65) & 9250 & 196 & 562 & 36 \\ \mathrm{H}(66) & 11984 & 760 & 1419 & 61 \\ \mathrm{H}(67) & 11993 & 196 & 1015 & 61 \\ \mathrm{H}(68) & 11315 & 282 & 1849 & 61 \\ \mathrm{H}(69) & 9627 & 1484 & -103 & 38 \\ \mathrm{H}(70) & 8321 & 1915 & -141 & 38 \\ \mathrm{H}(71) & 7632 & 893 & -818 & 72 \\ \mathrm{H}(72) & 7674 & 1388 & -1413 & 72 \\ \mathrm{H}(73) & 6360 & 1339 & -885 & 72 \\ \mathrm{H}(74) & 8908 & 2165 & 3748 & 46 \\ \mathrm{H}(75) & 7987 & 2487 & 2917 & 46 \\ \mathrm{H}(76) & 11244 & 2253 & 3303 & 75 \\ \mathrm{H}(77) & 10531 & 2810 & 3393 & 75 \\ \mathrm{H}(78) & 10332 & 2559 & 2449 & 75 \\ \mathrm{H}(79) & 9909 & 900 & 4095 & 43 \\ \mathrm{H}(80) & 10302 & 1503 & 4145 & 43 \\ \mathrm{H}(81) & 11766 & 763 & 3269 & 69 \\ \mathrm{H}(82) & 12593 & 1028 & 4180 & 69 \\ \mathrm{H}(83) & 12151 & 1366 & 3308 & 69\end{array}$

